\title{
Postsynaptic Density Scaffold SAP102 Regulates Cortical Synapse Development through EphB and PAK Signaling Pathway
}

\author{
Yasunobu Murata and Martha Constantine-Paton \\ McGovern Institute for Brain Research, Massachusetts Institute of Technology, Cambridge, Massachusetts 02139
}

Membrane-associated guanylate kinases (MAGUKs), including SAP102, PSD-95, PSD-93, and SAP97, are scaffolding proteins for ionotropic glutamate receptors at excitatory synapses. MAGUKs play critical roles in synaptic plasticity; however, details of signaling roles for each MAGUK remain largely unknown. Here we report that SAP102 regulates cortical synapse development through the EphB and PAK signaling pathways. Using lentivirus-delivered shRNAs, we found that SAP102 and PSD-95, but not PSD-93, are necessary for excitatory synapse formation and synaptic AMPA receptor (AMPAR) localization in developing mouse cortical neurons. SAP102 knockdown (KD) increased numbers of elongated dendritic filopodia, which is often observed in mouse models and human patients with mental retardation. Further analysis revealed that SAP102 coimmunoprecipitated the receptor tyrosine kinase EphB2 and RacGEF Kalirin-7 in neonatal cortex, and SAP102 KD reduced surface expression and dendritic localization of EphB. Moreover, SAP102 KD prevented reorganization of actin filaments, synapse formation, and synaptic AMPAR trafficking in response to EphB activation triggered by its ligand ephrinB. Last, p21-activated kinases (PAKs) were downregulated in SAP102 KD neurons. These results demonstrate that SAP102 has unique roles in cortical synapse development by mediating EphB and its downstream PAK signaling pathway. Both SAP102 and PAKs are associated with X-linked mental retardation in humans; thus, synapse formation mediated by EphB/SAP102/PAK signaling in the early postnatal brain may be crucial for cognitive development.

\section{Introduction}

Membrane-associated guanylate kinase (MAGUK) family scaffolds, including SAP102, PSD-95, PSD-93, and SAP97, are predominant components of the postsynaptic density (PSD) at excitatory synapses (Sheng and Hoogenraad, 2007). MAGUKs interact with a wide variety of proteins including receptors, adhesion, cytoskeletal, and signaling molecules, and serve an important role in structural and functional synaptic plasticity (Funke et al., 2005; Elias and Nicoll, 2007).

During postnatal brain development, the MAGUKs show different expression profiles. SAP102 is the dominant scaffold in the fetal and neonatal brain, while PSD-95 and PSD-93 are expressed increasingly with age (Sans et al., 2000; van Zundert et al., 2004; Petralia et al., 2005). In the rodent visual cortex, PSD-95 and

Received June 18, 2012; revised Jan. 28, 2013; accepted Feb. 3, 2013.

Author contributions: Y.M. and M.C.-P. designed research; Y.M. performed research; Y.M. analyzed data; Y.M. and M.C.-P. wrote the paper.

This work was supported by National Institutes of Health Grant 5R01EY014074-18 (M.C.P.). We thank Morgan Sheng (Genentech) for providing the PSD-93 expression plasmid and the anti-SAP97 antibody, Johannes Hell (University of California, Davis, Davis, CA) for providing the anti-SAP102 antibody, Michael Greenberg (Harvard Medical School, Boston, MA) for providing the anti-EphB2 antibody and the EphB2 expression plasmid, and Carlos Lois (University of Massachusetts, Worcester, MA) for providing the lentivirus plasmids. We thank Akira Yoshii and Andrew Bolton for critical reading and feedback on the manuscript.

The authors declare no competing financial interests.

Correspondence should be addressed to Yasunobu Murata, McGovern Institute for Brain Research, Massachusetts Institute of Technology, 77 Massachusetts Avenue, Building 46-4151, Cambridge, MA 02139. E-mail: ymurata@mit.edu.

DOI:10.1523/JNEUROSCI.2896-12.2013

Copyright $\odot 2013$ the authors $\quad 0270-6474 / 13 / 335040-13 \$ 15.00 / 0$
SAP102 are rapidly increased at synapses after eye opening, suggesting crucial roles of MAGUKs in experience-dependent cortical development (Yoshii et al., 2003). In addition, MAGUKs show different functional properties, such as synaptic versus extrasynaptic localization and preferential interaction with GluN2A and GluN2B subunits of the NMDA receptor (NMDAR) (van Zundert et al., 2004; Zheng et al., 2011). However, whether each MAGUK has a distinct role in early cortical synapse development remains largely unknown.

The importance of SAP102 function in brain development is demonstrated by the fact that SAP102 mutations have been identified in human patients with nonsyndromic X-linked mental retardation (XLMR) (Tarpey et al., 2004; Zanni et al., 2010). Human patients with inherited forms of mental retardation often show abnormal dendritic spine morphology (Ramakers, 2002). Dendritic spines are rich in actin filaments, and small GTPbinding protein (Rac/Rho/cdc42) signaling is a key determinant for actin cytoskeleton dynamics. Among many upstream modulators of Rac signaling, the receptor tyrosine kinase EphB and the neuronal Rac1 GDP/GTP exchange factor (GEF) Kalirin-7 have been well characterized for their roles in synaptogenesis and spine morphogenesis (Penzes and Jones, 2008; Klein, 2009; Lai and Ip, 2009; Ma, 2010). Several proteins in the Rac signaling pathway, including downstream p21-activated kinase 3 (PAK3), have been identified as causal genes for mental retardation, implicating Rac and PAK signaling in the etiology of neurodevelopmental disorders (Humeau et al., 2009; Nadif Kasri et al., 2009; Boda et al., 2010). 
Here we describe the developmental role of SAP 102 in cortical synapse formation and maturation. Using lentivirus-mediated RNA interference, we found that SAP102 and PSD-95 play important roles in excitatory synaptogenesis and glutamate receptor trafficking in young cortical neurons. Interestingly, only SAP102 knockdown (KD) increased long dendritic filopodia as is often observed in model mice and human patients with XLMR (Ramakers, 2002). Our further analysis revealed that SAP102 is in a complex with EphB2 and Kalirin-7, and that their downstream PAK activity is suppressed in SAP102 KD neurons. Although MAGUKs are highly similar in amino acid sequence, they show differential association with neuropsychiatric diseases. Our study documents functional association between two causal genes for XLMR: SAP102 and PAK3, and shows that SAP102 has critical roles in early cortical synapse development.

\section{Materials and Methods}

Construction of lentiviral shRNA vector. The lentiviral shRNA plasmid pLL 3.7 (gift from Carlos Lois, University of Massachusetts, Worcester, MA) was modified to enhance neuronal expression of GFP or tdTomato by replacing the CMV promoter with the human synapsin 1 promoter (Rubinson et al., 2003; Dittgen et al., 2004). The following oligonucleotides encoding short hairpin RNAs (shRNAs) were inserted under the U6 promoter between HpaI and XhoI sites. For all three MAGUKs, the two shRNAs were equally effective and used interchangeably in some experiments. All these shRNAs were designed to knock down all known splice variants. Scrambled shRNA sequences in which nucleotides were shuffled were used as negative controls as follows: PSD95 shRNA 1: 5' -tGTCAGACGGTCACGATCATttcaagagaA TGATCGTGACCGTCTGACttttttc-3' $5^{\prime}$-tcgagaaaaaaGTCAGACGG TCACGATCATtctcttgaaATGATCGTGACCGTCTGACa-3'; PSD95 scrambled shRNA 1: 5' -tGTCAGACGTGCACGATCATttcaagagaATGAT CGTGCACGTCTGACttttttc-3' $5^{\prime}$-tcgagaaaaaaGTCAGACGTGCACGAT CATtctcttgaaATGATCGTGCACGTCTGACa-3'; PSD95 shRNA 2: '-tGAT GAAGACACGCCCCCTCttcaagagaGAGGGGGCGTGTCTTCATCtttttt c-3' $5^{\prime}$-tcgagaaaaaaGATGAAGACACGCCCCCTCtctcttgaaGAGGGGGCG TGTCTTCATCa-3'; PSD95 scrambled shRNA 2: 5' -tGCCCTACCACCGA GGTCAAttcaagagaTTGACCTCGGTGGTAGGGCttttttc-3' 5' -tcgagaaaaa aGCCCTACCACCGAGGTCAAtctcttgaaTTGACCTCGGTGGTAGGGC a-3'; SAP102 shRNA 1: 5'-tGCCAGTGACACGACAAGAAttcaagagaTTC TTGTCGTGTCACTGGCttttttc-3' 5' -tcgagaaaaaaGCCAGTGACACGAC AAGAAtctcttgaaTTCTTGTCGTGTCACTGGCa-3'; SAP102 scrambled shRNA 1: 5'-tGCCAGTGAACCGACAAGAAttcaagagaTTCTTGTCGGTT CACTGGCtttttc- $3^{\prime} 5^{\prime}$-tcgagaaaaaGCCAGTGAACCGACAAGAAtctcttga aTTCTTGTCGGTTCACTGGCa-3'; AP102 shRNA 2: 5' -tGGTTAAGTG ACGATTATTAttcaagagaTAATAATCGTCACTTAACCtttttc- $3^{\prime} 5^{\prime}$-tcgaga aaaaGGTTAAGTGACGATTATTAtctcttgaaTAATAATCGTCACTTAAC Ca-3'; SAP102 scrambled shRNA 2: 5' -tGGTTAAGTAGCGATTATTAttca agagaTAATAATCGCTACTTAACCttttttc-3' $5^{\prime}$-tcgagaaaaaaGGTTAAGTA GCGATTATTAtctcttgaaTAATAATCGCTACTTAACCa-3'; PSD-93 shRNA 1: 5' -tGCCAAAGCGTGACTACGAAGTcgaaACTTCGTAGTCA CGCTTTGGCtttttc- $3^{\prime} 5^{\prime}$-tcgagaaaaaaGCCAAAGCGTGACTACGAAGTtt cgACTTCGTAGTCACGCTTTGGCa-3'; PSD-93 scrambled shRNA 1: 5' tGCCAAAGCGGTACTACGAAGTcgaaACTTCGTAGTCACGCTTTGG Ctttttc- $3^{\prime}$ 5'-tcgagaaaaaaGCCAAAGCGGTACTACGAAGTttcgACTTCG TAGTCACGCTTTGGCa-3'; PSD-93 shRNA 2: 5' -tGTGAATAAACTGT GTGATAttcaagagaTATCACACAGTTTATTCACtttttc- $3^{\prime} 5^{\prime}$-tcgagaaaaaaG TGAATAAACTGTGTGATAtctcttgaaTATCACACAGTTTATTCACa-3'; PSD-93 scrambled shRNA 2: 5' -tGTGAATAACATGTGTGATAttcaagagaT ATCACACATGTTATTCACtttttc- $3^{\prime}$ 5' ${ }^{\prime}$-tcgagaaaaaGTGAATAACATGT GTGATAtctcttgaaTATCACACATGTTATTCACa-3'.

Production of lentivirus. Lentiviruses were produced as previously described (Lois et al., 2002). Briefly, human embryonic kidney $293 \mathrm{~T}$ (HEK293T) cells were transfected using Lipofectamine 2000 (Invitrogen) with lentiviral shRNA plasmid, $\Delta 8.9$ plasmid and vesicular stomatitis virus G-protein plasmid at 20,15, and $10 \mu \mathrm{g}$ of DNA per $15 \mathrm{~cm}$ plate. Forty-eight hours after transfection, culture medium was collected and centrifuged at $2000 \times g$ for $10 \mathrm{~min}$. Supernatants were filtered through a
$0.45 \mu \mathrm{m}$ filter and centrifuged at $83,000 \times g$ for $1.5 \mathrm{~h}$, and the resulting pellets were resuspended in PBS. The titer of lentivirus was between $5 \times$ $10^{4}$ to $1 \times 10^{5}$ infectious units per microliters.

Evaluation of efficacy and specificity of shRNA sequences in HEK293T cells. HEK293T cells were cotransfected using Lipofectamine 2000 with the shRNA plasmids as well as the expression plasmids of SAP102-GFP, PSD-95-GFP, and myc-PSD-93 (gift from Morgan Sheng, Genentech). Forty-eight hours after transfection, cell lysates were prepared and analyzed by Western blotting.

Primary cortical neuron culture. All manipulations were performed in accord with the guidelines of the Massachusetts Institute of Technology Institutional Animal Care and Use Committee. Primary cortical neuron cultures were prepared from male and female embryonic day 15 (E15) mice or E18 rats (Banker and Goslin, 1991; Yoshii and ConstantinePaton, 2007). Most cultures were derived from mouse occipital cortices and a few were from mouse or rat whole cortex. We detected no difference in the results reported between mice and rats nor between whole and occipital cortex culture. Cortices were dissected out and treated with papain (Worthington) and DNaseI (Sigma) for $10 \mathrm{~min}$ at $37^{\circ} \mathrm{C}$ and triturated with a fire-polished Pasteur pipette. Cells were plated at the density of $5 \times 10^{4}$ cells $/ \mathrm{cm}^{2}$ on coverslips or plastic dishes that were precoated with $\alpha$-laminin and poly-D-lysine, and cultured in Neurobasal medium supplemented with B-27 and $200 \mathrm{~mm}$ glutamine. Cultured cortical neurons were infected with lentiviruses at day in vitro (DIV) 2 or DIV 14. The ratios of infectious viral particles to cells were 2 for biochemical analyses whereas 0.1 for immunocytochemical analyses for ease of visualization of dendritic morphology.

For activation of EphB signaling, EphrinB2-Fc or Fc recombinant proteins (R\&D Systems) were dimerized with anti-human Fc antibody (Jackson ImmunoResearch Laboratories) at a 2:1 ratio in Neurobasal medium at room temperature for $1 \mathrm{~h}$. Dimerized ephrinB2-Fc or Fc were bath applied to cultured neurons at $500 \mathrm{ng} / \mathrm{ml}$ and incubated at $37^{\circ} \mathrm{C}$ for $1 \mathrm{~h}$.

Preparation of homogenate, synaptosomal membrane, and PSD fraction. PSD and synaptosomal fractions were isolated from cultured cortical neurons as previously described (Ehlers, 2003). All buffers contained a protease inhibitor cocktail (Roche) and all procedures were performed on ice or at $4^{\circ} \mathrm{C}$. Briefly, cultured neurons were homogenized in $0.32 \mathrm{M}$ sucrose and 4 mM HEPES, pH 7.4, and centrifuged at $1000 \times g$ to remove the pelleted nuclear fraction and debris. The resulting supernatant was collected as homogenate. Homogenate was centrifuged at $10,000 \times g$ for $15 \mathrm{~min}$; the supernatant was collected as cytosolic fraction and the pellet was collected as crude membrane fraction. The membrane fraction pellet was lysed by hypo-osmotic shock in 4 mM HEPES, pH 7.4, and centrifuged at 25,000 $\times g$ for $20 \mathrm{~min}$. The resulting pellet was resuspended in 50 mM HEPES, pH 7.4, and 2 mM EDTA, and collected as synaptosomal membrane fraction. To obtain PSD fractions, Triton X-100 (final concentration $0.5 \%$ ) was added to the solution, rotated for $15 \mathrm{~min}$, and centrifuged at $32,000 \times g$ for $20 \mathrm{~min}$ to obtain the pellet. The pellet was resuspended in ice-cold $50 \mathrm{~mm}$ HEPES, pH 7.4, and 2 mM EDTA, then Triton X-100 (final concentration $0.5 \%$ ) was added to the solution, rotated for $15 \mathrm{~min}$, and centrifuged at 200,000 $\times \mathrm{g}$ for $20 \mathrm{~min}$. The pellet was resuspended in $50 \mathrm{~mm}$ HEPES, $\mathrm{pH}$ 7.4, and $2 \mathrm{~mm}$ EDTA and collected as PSD fraction.

Surface biotinylation assay. All buffers contained a protease inhibitor cocktail and all procedures were performed on ice or at $4^{\circ} \mathrm{C}$. Cultured neurons were washed with ice-cold PBS and incubated with $1.5 \mathrm{mg} / \mathrm{ml}$ sulfo-NHS-biotin (Thermo Scientific) in PBS for $30 \mathrm{~min}$. After washing $(3 \times)$ with $50 \mathrm{~mm}$ glycine in PBS, neurons were lysed with radioimmunoprecipitation assay (RIPA) buffer (50 mm Tris, pH 7.8, $150 \mathrm{~mm} \mathrm{NaCl}$, $1 \%$ Triton X-100, $0.5 \%$ deoxycholate, $0.1 \%$ SDS), sonicated, and centrifuged at 10,000 $\times g$ for $10 \mathrm{~min}$ to obtain the cell lysates. Cell lysates (300 $\mu \mathrm{g}$ ) were incubated with $70 \mu \mathrm{l}$ of NeutrAvidin agarose resins (Thermo Scientific) overnight. Proteins unbound to NeutrAvidin agarose resins were collected as cytosolic fractions. After washing $(3 \times)$ with RIPA buffer, bound proteins were eluted with SDS sample buffer, collected as surface fraction, and analyzed by Western blotting.

Immunoprecipitation. All buffers contained a protease inhibitor cocktail and all procedures were performed on ice or at $4^{\circ} \mathrm{C}$. Visual cortical 
tissue from postnatal 14 (P14) or adult male and female rats were homogenized and centrifuged at $710 \times g$ for $10 \mathrm{~min}$ to remove debris and nuclei. The resulting supernatants were suspended in lysis buffer (1\% Triton X-100 in 50 $\mathrm{mm}$ Tris, $\mathrm{pH} 7.5,150 \mathrm{~mm} \mathrm{NaCl}$ ), rotated for $1 \mathrm{~h}$, and centrifuged at $10.000 \times$ $g$ at for $10 \mathrm{~min}$. Since the PSD is not completely formed at P14 in the visual cortex, most PSD proteins, including SAP102 and PSD-95, were solubilized under this condition. The resulting supernatants were mixed with antibodybound Dynabeads, protein G (Invitrogen), and incubated overnight. After washing $(3 \times)$ with lysis buffer, bound proteins were eluted with SDS sample buffer and analyzed by Western blotting.

For coimmunoprecipitation assay in HEK293 cells, cells were cotransfected with SAP102-GFP and GluN2B or flag-EphB expression plasmids using Lipofectamine 2000. After 48 h of incubation, harvested cells were lysed with $1 \%$ Triton X-100 in PBS and centrifuged at $10.000 \times g$ for 10 min. The resulting supernatants were mixed with Dynabeads protein $G$ bound with the anti-GFP antibody and incubated overnight. After washing $(3 \times)$ with $1 \%$ Triton X-100 in PBS, bound proteins were eluted with SDS sample buffer and analyzed by Western blotting.

Antibodies used for Western blotting. The following antibodies were used: anti-PSD-95 (NeuroMab, 75-028), anti-SAP102 (gift from Johannes Hell, University of California, Davis, Davis, CA; NeuroMab, 75058), anti-PSD-93 (NeuroMab, N18/30), SAP97 (gift from Morgan Sheng, Genentech; NeuroMab, 75-030; Affinity BioReagent, PA1-741), anti-pan-MAGUK (mouse, NeuroMab, K28/86), anti-tubulin $\beta$ III (Covance, MMS-435P; Abcam, ab6046), anti- $\beta$ actin (Sigma, AC-40; Abcam, ab8226), anti-GluN1 (BD Biosciences, 54.1 or NeuroMab, N308-48), anti-GluN2A (Millipore, 04-901 and 05-901R), antiGluN2B (NeuroMab, 75-097 and 75-101), anti-GluA1 (Millipore, AB1504), anti-GluA2 (Millipore, MAB397; NeuroMab, 75-002), antitransferrin receptor (Invitrogen, 13-6800), anti-EphB2 (gift from Michael Greenberg, Harvard Medical School, Boston, MA), anti-FLAG (Sigma, F1804), anti-GFP (Abcam, ab290), anti-pan-Kalirin (Millipore, 07-122), anti-PAK1 (Cell Signaling Technology, 2602), anti-PAK3 (Cell Signaling Technology, 2609), anti-phospho PAK 144/141/139 (Cell Signaling Technology, 2606), horseradish peroxidase-conjugated anti-mouse, anti-rabbit and anti-rat secondary antibodies (Jackson ImmunoResearch and Pierce).

We tested three different anti-SAP97 antibodies that have been shown to work in hippocampus (Nakagawa et al., 2004; Schlüter et al., 2006), but none of these antibodies was able to detect clear bands on immunoblots of young cultured cortical neurons (data not shown).

Quantification of band intensities in Western blotting was performed by ImageJ software. The signal intensities of bands were normalized to loading controls. All data presented are from at least three independent experiments described in the text.

Immunocytochemistry. Cultured neurons were fixed with $4 \%$ paraformaldehyde in PBS for $10 \mathrm{~min}$ and permeabilized and blocked with 5\% goat serum and $0.3 \%$ Triton X-100 in PBS for $1 \mathrm{~h}$. After incubation with primary antibodies for overnight at $4^{\circ} \mathrm{C}$ and with secondary antibodies for $1 \mathrm{~h}$ at room temperature, coverslips were mounted with Fluoromount-G (electron microscopy). The following primary antibodies or fluorescent dyes were used for immunocytochemistry: anti-GFP (Nacalai, GF090R, 1:5000), anti-Bassoon (Stressgen, VAM-PS003E, 1:1000), anti-VGLUT1 (Synaptic Systems, 135 303, 1:1000), anti-VGAT (Synaptic Systems, 131 011, 1:1000), ephrinB2-Fc (R\&D Systems, $5 \mu \mathrm{g} / \mathrm{ml}$ ) (Tolias et al., 2007), and phalloidinAlexa 543 (Invitrogen, 1:500). All secondary antibodies, including goat antimouse, anti-rabbit, anti-rat. or anti-human conjugated with Alexa 488, Alexa 543, or Alexa 633 (Invitrogen), were used at 1:1000.

The confocal $z$-stack images were taken with a Nikon PCM 2000 with a $60 \times$ oil objective (NA 1.4) at $0.5 \mu \mathrm{m} z$-interval using the same laser intensity and parameters for each experiment. Figures present a projection from these confocal $z$-stacks. Image analysis was performed with ImageJ software. Puncta with $>4$ pixels $\left(0.16 \mu \mathrm{m}^{2}\right)$ associated with GFP or tdTomato-expressing dendrites and dendritic protrusions were analyzed after thresholding at a constant value for each experiment. Only phalloidin signals inside GFP-positive dendrites and dendritic protrusions were analyzed. Dendritic protrusion length was defined as the distance from the edge of the dendritic shaft to the tip of the protrusion. Image acquisition and analysis were performed in a blind manner.
Statistical analysis. All statistical analyses were performed with SPSS software using either paired $t$ tests or ANOVA with a post hoc Tukey's or Bonferroni test. All data are presented as mean \pm SEM. A value of $p<$ 0.05 was accepted as statistically significant. Only significant ANOVA results are shown with asterisks in the figures: ${ }^{\star} p<0.05,{ }^{* *} p<0.01$. In all experiments, no significant differences were found between neurons infected with scrambled control shRNA lentiviruses and GFP (only) lentiviruses. Therefore, the fluorescently tagged lentiviruses are shown as the control in most experiments.

\section{Results}

\section{SAP102 and PSD-95 are major postsynaptic scaffolds of} glutamate receptors in developing visual cortex

We examined developmental expression changes of MAGUKs in vitro by analyzing cortical culture homogenates prepared at different ages from DIV 10-26. Quantitative immunoblot analyses showed that SAP102 appeared from an early developmental stage whereas PSD-95 gradually increased during development. PSD-93 remained at relatively low expression levels until DIV 18 and then rapidly increased (Fig. 1A,B). Consequently, because our focus was on the developmental function of these MAGUKs, we define DIV 14 synapses as "developing synapses" for most subsequent culture experiments: This stage in culture represents a time point where cortical synapses have begun to mature but have not yet attained their full complement of MAGUKs. These developmental expression profiles of SAP102, PSD-95, and PSD-93 in vitro are similar to our observations in the rodent visual cortex in vivo (Y. Murata and M. Constantine-Paton, unpublished observations).

We used lentivirus-delivered shRNAs to study the roles of each MAGUK in cortical synapse development. We designed two different shRNA sequences for each MAGUK (SAP102, PSD-95, and PSD-93). Since two different shRNA sequences showed very similar results, we did not show the results of second shRNA in some subsequent experiments to avoid repetition. As negative controls, we created corresponding scrambled shRNA sequences within which nucleotides were shuffled. Target specificity of each shRNA sequence was verified in HEK293 cells using cotransfection of shRNA constructs and expression plasmids of SAP102, PSD-95, and PSD-93, followed by Western blotting. Each shRNA sequence specifically knocked down the target protein and had no effect on expression of other MAGUKs (data not shown). Next we validated efficacy and specificity of MAGUK KD in cultured cortical neurons (Fig. 1C). Neurons were infected at DIV 2 with lentivirus-expressing shRNA or the control scrambled shRNA (scr.sh). In addition, GFP lentivirus control infections were included for each experiment. Knockdown was effective by $7 \mathrm{~d}$ after lentivirus infection for all shRNAs and lasted for $>4$ weeks (data not shown). Scrambled control shRNAs did not affect expression of any MAGUKs (Fig. 1C), and were identical on Western blotting to the lentivirus control (GFP lentivirus) for each MAGUK (data for PSD-95 and PSD-93 not shown). Notably, knockdown of each MAGUK caused upregulation of other MAGUKs (Fig. 1E); a probable compensatory mechanism that has been previously reported in knock-out mice (Elias et al., 2006; Cuthbert et al., 2007).

Next we analyzed whether knockdown of each MAGUK affects the total amount of all MAGUKs by using an anti-pan-MAGUK antibody recognizing SAP102, PSD-95, PSD-93, and SAP97. At DIV 14, SAP102 shRNA and PSD-95 shRNA decreased the total MAGUK expression level by 34 and 38\%, respectively, whereas PSD-93 shRNA had no significant effects on the total MAGUK level, suggesting that SAP102 and PSD-95 are the two abundant MAGUKs in developing cortical neurons. No clear band was detected with the 
A

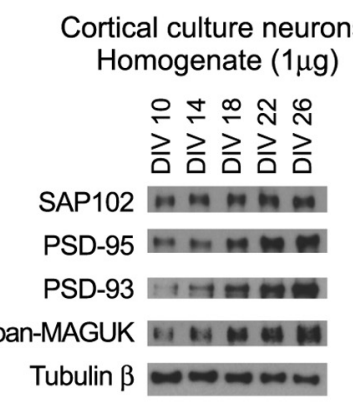

C
DIV 14: Homogenate [ shRNA lentivirus from DIV 2 ]

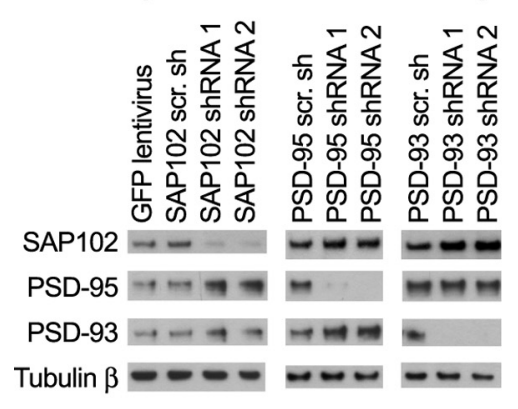

B

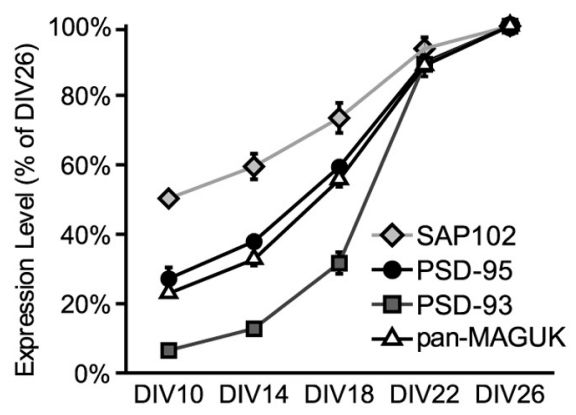

D

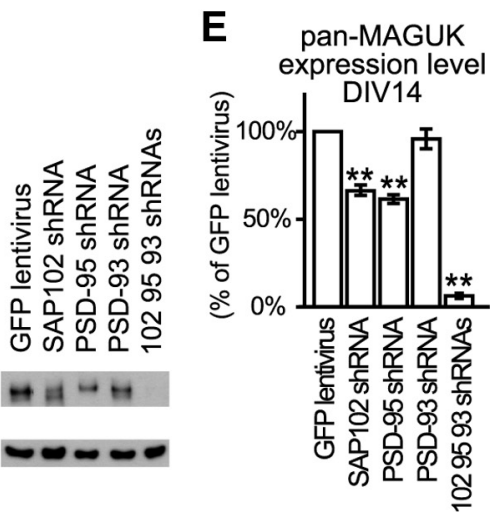

Figure 1. SAP102 and PSD-95 are the two major postsynaptic MAGUKs in developing cortical neurons. $A, B$, Different developmental expression profiles of SAP102, PSD-95, and PSD-93 in cultured cortical neurons. Homogenates $(1 \mu \mathrm{g})$ from cultured cortical neurons at DIV $10,14,18,22$, and 26 were analyzed by quantitative Western blotting. These blots were quantified and plotted as a percentage of each MAGUK's level at DIV 26. Total MAGUK levels were analyzed with an anti-pan-MAGUK antibody recognizing SAP102, PSD-95, PSD-93, and SAP97. SAP102 was expressed at relatively high levels from the earliest developmental stage examined, and PSD-95 levels were gradually increased whereas PSD-93 remained at relatively low levels until DIV 18 and increased very rapidly between DIV 18 and 22. No clear band was detected with the anti-SAP97 antibodies (see Materials and Methods). $(n=6$ immunoblots from three separate sets of cultures, data are presented as mean \pm SEM.) C, Lentivirus-delivered shRNAs effectively knocked down specific MAGUKs in DIV 14 cultured cortical neurons. Neurons were infected with lentiviruses expressing shRNA or the corresponding control scrambled shRNA (scr.sh) at DIV 2. For each MAGUK two different shRNA and scrambled shRNA lentiviruses were used. The shRNA specifically reduced the target protein to a point that it was undetectable or barely detectable on immunoblots, and the knockdown lasted for at least four weeks in the cultures (data not shown). As illustrated for SAP102, the GFP lentivirus control was not different from the SAP102 scr.sh. This was tested in one-way ANOVAs (data not shown). The same ANOVA test was used for GFP lentivirus or tdTomato lentivirus and the scrambled shRNAs for each MAGUK in all subsequent experiments and no significant differences were found. Therefore, GFP or tdTomato lentivirus is shown as the control in most experiments. D, E, SAP102 shRNA and PSD-95 shRNA, but not PSD-93 shRNA, significantly decreased the amount of total MAGUK expression levels in developing cultured cortical neurons. Total MAGUK levels were analyzed with an anti-pan-MAGUK antibody recognizing SAP102, PSD-95, PSD-93, and SAP97. Knockdown of SAP102 or PSD-95, but not PSD-93, significantly reduced the total MAGUK levels, suggesting SAP102 and PSD-95 are predominant MAGUKs in these developing neurons. Triple knockdown of SAP102, PSD-95, and PSD-93, was achieved by coinfection with a lentivirus against each MAGUK. Triple KD decreased the total MAGUK levels by $95 \%$, indicating that the last member of MAGUKs, SAP97, is far less abundant than SAP102, PSD-95 or PSD-93 in young cortical neurons. ( $n=6$ immunoblots from three separate sets of cultures.) One-way ANOVA tests were used in this and all subsequent data presented. Data are presented as mean \pm SEM, and asterisks are $p$ values comparing to the shRNA lentivirus infected neurons to the GFP lentivirus infected control neurons; ${ }^{*} p<$ $0.05,{ }^{* *} p<0.01$.

anti-SAP97 antibodies (see Materials and Methods). Furthermore, triple knockdown of SAP102, PSD-95, and PSD-93 decreased the total MAGUK level by $>95 \%$ (Fig. $1 D, E$ ) suggesting that SAP97 is a minor MAGUK in young cortical neurons.

\section{SAP102 and PSD-95, but not PSD-93, are necessary for excitatory synaptogenesis and synaptic AMPA receptor localization}

Among MAGUKs, PSD-95 has been extensively studied and its roles in excitatory synapse formation have been previously documented (El-Husseini et al., 2000; Gerrow et al., 2006). To examine and compare the function of each MAGUK in cortical synapse development, we used two different shRNAs for each MAGUK to knock down either SAP102, PSD-95, or PSD-93 in cultured cortical neurons at DIV 2 and performed immunocyto- chemistry with a presynaptic marker Bassoon at DIV 14 (Fig. 2A). The effects of scrambled shRNA lentiviruses and GFP lentivirus were not significantly different in all experiments; therefore, GFP lentivirus was used as the control in all figures. SAP102 shRNAs and PSD-95 shRNAs, but not PSD-93 shRNAs, significantly reduced the density of Bassoon puncta associated with GFP-positive lentivirusinfected dendrites (Fig. 2B). Control shRNAs had no effect on the density of Bassoon puncta (Fig. 2B). We confirmed that the two shRNA sequences for each MAGUK resulted in comparable reductions in Bassoon puncta density to ensure the validity of the knockdown effects.

Next, we examined the role of MAGUKs on excitatory and inhibitory synapse formation by immunostaining cortical neurons with an excitatory presynaptic marker: anti-vesicular glutamate transporter 1 (VGLUT1) and with an inhibitory presynaptic marker: anti-vesicular GABA transporter (VGAT) (Fig. 2C). Knockdown of SAP102 or PSD-95, but not PSD-93, caused a selective decrease in the density of VGLUT1 in DIV 14 cortical neurons (Fig. $2 D)$. However, the densities of VGAT puncta were not significantly altered by any of the MAGUK KDs (Fig. 2D).

The function of MAGUKs in glutamate receptor trafficking has been characterized in hippocampal neurons. Manipulation of SAP102 affects AMPA receptor (AMPAR) currents during the early postnatal period while PSD-95 and PSD-93 regulate AMPAR trafficking upon maturation (Elias and Nicoll, 2007). To distinguish the role of each MAGUK in synaptic localization of glutamate receptors in developing cortical neurons, we analyzed the levels of NMDAR and AMPAR subunits in the PSD fraction at DIV 14 (Fig. 2F, G). The quality of PSD isolation was validated by the absence of Synaptophysin, a presynaptic vesicle related protein, in the PSD fraction (Fig. $2 H$ ). Knockdown of SAP102 or PSD-95, but not PSD-93, reduced the amount of the GluA1 (GluR1) and GluA2 (GluR2) subunit of the AMPAR in PSD fractions. There were not large differences in GluA1 and GluA2. We therefore used GluA2 as a proxy for AMPARs for the rest of the study. None of the MAGUK knockdowns significantly altered NMDAR subunit levels in the PSD fractions. These findings are consistent with most previous electrophysiological studies showing MAGUK knockdown or knock-outs have greater effects on AMPAR than NMDAR currents in hippocampal synapses (Béique et al., 2006; Elias et al., 2006, 2008; Schlüter et al., 2006; Ehrlich et al., 2007; Futai et al., 2007), suggesting a compensation mechanism to maintain NMDAR levels at synapses when a MAGUK is absent. For example, in SAP102 knock-out (KO) mice, an increased amount of PSD-95 associates with GluN1 (Cuthbert et al., 2007). Given that triheteromeric GluN1-GluN2AGluN2B NMDARs are present at synapses (Gray et al., 2011; Rauner 

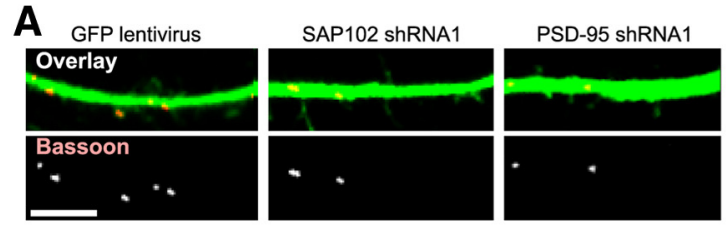

SAP102 ShRNA2

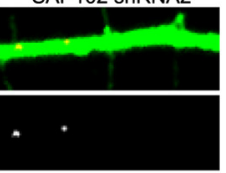

C
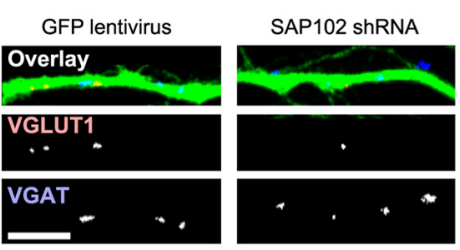

DIV 14: Homogenate

$\mathbf{E}$

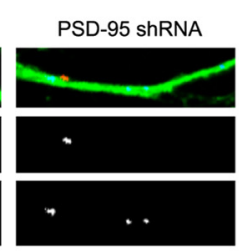

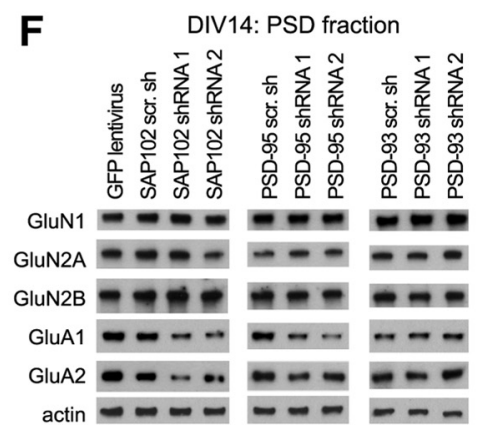
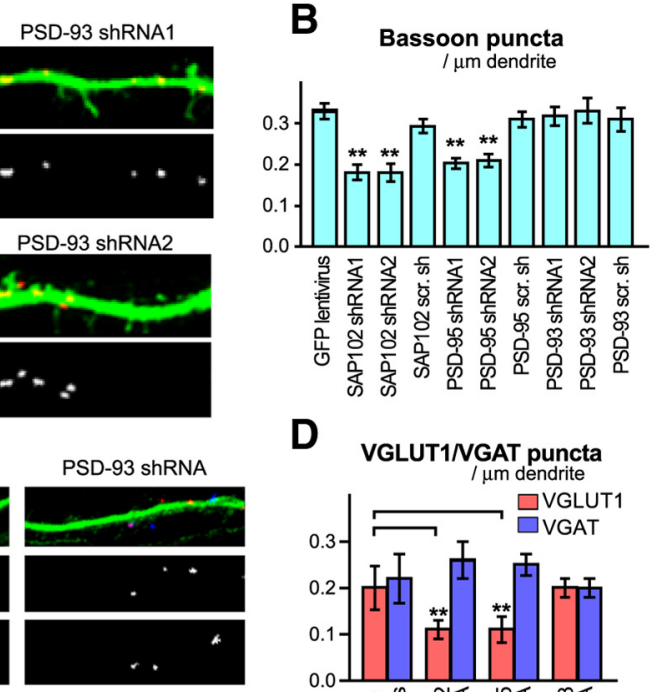

D

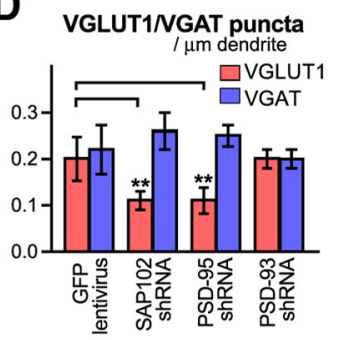

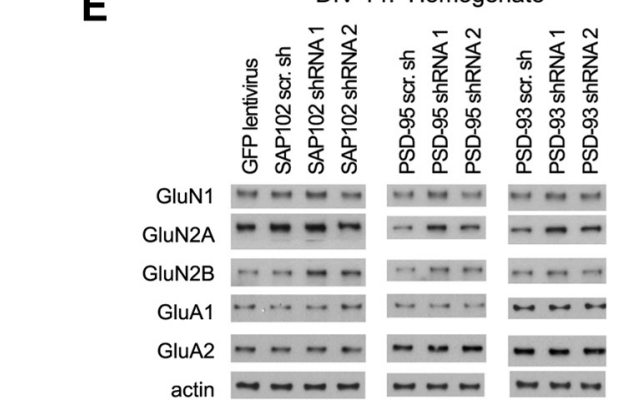
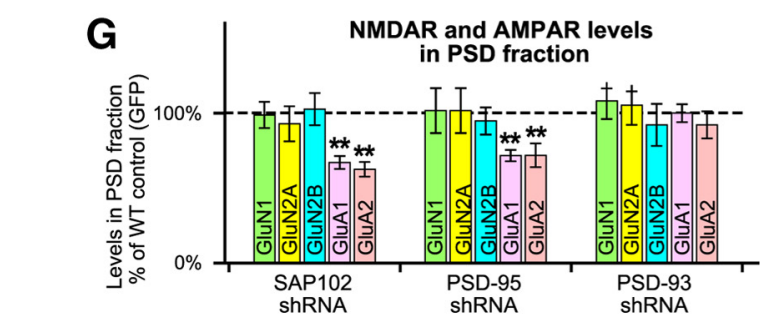

Figure 2. SAP102 and PSD-95 play important roles in excitatory synapse formation and synaptic AMPAR localization in developing cortical neurons. A, B, SAP102 shRNAs and PSD-95 shRNAs, but not PSD-93 shRNAs, reduced puncta of the presynaptic marker Bassoon. Cortical neurons were infected with lentiviruses containing shRNA or scrambled shRNA (scr.sh) at DIV 2 and immunostained with an anti-Bassoon antibody at DIV 14. The density of Bassoon puncta on dendrites showed a 40\% decrease in SAP102 KD and PSD-95 KD neurons compared with their appropriate scrambled control, but remained constant in PSD-93 KD neurons. Note that similar results were obtained from two different shRNA lentiviruses for each MAGUK, which ensure the validity of the knockdown effects. Since two different shRNA sequences showed very similar results, we did not show the results of second shRNA in some subsequent experiments to avoid repetition. Scrambled shRNAs had no significant effect on the densities of Bassoon puncta. Scale bar, $5 \mu \mathrm{m}$. ( $n=>15$ dendrites from 8 neurons). C, D, SAP102 shRNA and PSD-95 shRNA reduced the excitatory presynaptic marker VGLUT1. DIV 14 neurons were doubly immunostained with anti-VGLUT1 (excitatory presynaptic marker, red) and anti-VGAT (inhibitory presynaptic marker, blue). The densities of VGLUT1 puncta were significantly reduced in SAP102 KD and PSD-95 KD neurons whereas the density of VGAT puncta was not significantly altered in any of MAGUK KDs. Scale bar, $5 \mu \mathrm{m}$. ( $n=>15$ dendrites from 8 neurons). E-G, SAP102 and PSD-95 play major roles in maintaining synaptic AMPAR levels in developing cortical neurons. The PSD fraction was prepared from DIV 14 cortical cultures and analyzed by Western blotting. The levels of NMDAR and AMPAR subunits in homogenate were not altered by any of MAGUK KDs (E). The levels of glutamate receptor subunits in the PSD fraction $(\boldsymbol{F})$ were plotted as a histogram (G). SAP102 shRNAs and PSD-95 shRNAs, but not PSD-93 shRNAs, significantly reduced the levels of the GluA1 and GluA2 AMPAR subunits in the PSD fraction. None of MAGUK KDs changed the levels of NMDAR subunits in the PSD fraction, thereby indicating a greater effect of MAGUK KDs on AMPARs than NMDARs at the PSD. ( $n=6$ immunoblots from 3 independent sets of cultures.) $\boldsymbol{H}$, The subcellular fractions from DIV 14 cultured cortical neurons, including homogenate, cytosol, crude membrane, synaptosome, and PSD fraction, were analyzed by Western blotting. SAP102, PSD-95, GluN1, and GluA2 were strongly enriched in the PSD fraction. The absence of Synaptophysin, presynaptic vesicle related protein, in the PSD fraction validates the isolation of PSD fraction.

and Köhr, 2011), such a compensation mechanism could mask the effects of MAGUK KD on NMDAR subunits at the PSD. There was no decrease in the total expression level of NMDAR and AMPAR subunits after any MAGUK KD (Fig. 2E). Therefore, the results above indicate that in developing cortical neurons, SAP102 and PSD-95, but not PSD-93, are necessary for excitatory synapse development including both presynaptic specialization and AMPAR incorporation at the PSD.
Increased long-thin dendritic filopodia in SAP102

KD neurons

Next we examined whether SAP102 KD or PSD-95 KD affects the morphology of dendritic protrusions. We infected cultured cortical neurons with lentivirus-expressing GFP only, SAP102 shRNA1, SAP102 shRNA2, PSD-95 shRNA1, or PSD-95 shRNA2 at DIV 2. We then fixed the cultures and imaged them with confocal microscopy at DIV 14 (Fig. 3A). The GFP lentivirus control neurons had 


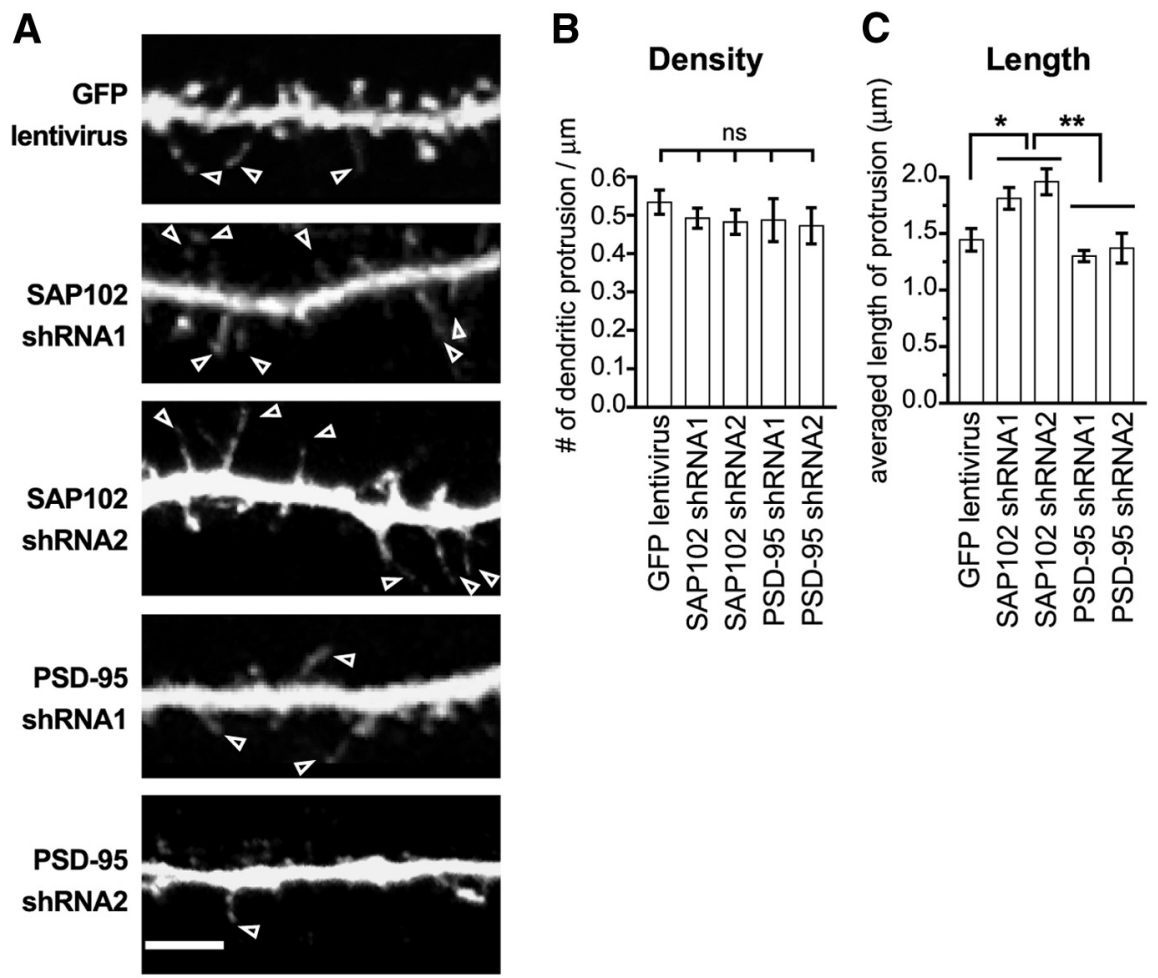

Figure 3. SAP102 shRNA increases dendritic filopodia length. $\boldsymbol{A}$, Representative confocal images of dendritic protrusions in control GFP lentivirus, SAP102 KD, and PSD-95 KD neurons at DIV 14. Cultured cortical neurons were infected with GFP control, SAP102 shRNA1, SAP102 shRNA2, PSD-95 shRNA1, or PSD-95 shRNA2 lentivirus at DIV 2. Neurons were fixed and imaged at DIV 14. Scale bar, $5 \mu \mathrm{m}$. $\boldsymbol{B}$, The density of dendritic protrusions was not significantly altered in SAP102 KD and PSD-95 KD neurons ( $n=10$ dendrites from 5 neurons. The number of dendritic protrusions per micrometer dendrite is presented as mean \pm SEM.) $\boldsymbol{C}$, The average length of dendritic protrusions was significantly increased in SAP102 KD neurons reflecting the prevalence of long dendritic filopodia caused by SAP102 shRNAs. The average length of dendritic protrusions in PSD-95 KD was slightly shorter compared with GFP lentivirus-infected neurons because of the prevalence of small stubby protrusions in PSD-95 KD neurons, but the difference did not reach statistical significance. ( $n=10$ dendrites from 5 neurons.)

normal mushroom-shaped spines and a few filopodia. However, long dendritic filopodia were prevalent in SAP102 KD neurons, whereas small stubby protrusions are prevalent in PSD-95 KD neurons. The density of dendritic protrusions was not significantly different between GFP lentivirus control, SAP102 KD, and PSD-95 KD neurons (Fig. 3B). However, the average length of dendritic protrusion was significantly increased in SAP102 KD neurons compared with GFP control and PSD-95 KD neurons, reflecting the prevalence of long dendritic filopodia caused by SAP102 shRNAs (Fig. 3C). Significantly, SAP102 mutations have been found in human patients with mental retardation (Tarpey et al., 2004; Zanni et al., 2010), and this aberrant protrusion morphology is a hallmark of pathology in inherited forms of mental retardation (Ramakers, 2002).

SAP102 is in a complex with EphB2 in early postnatal cortex and regulates surface and dendritic expression of EphB2

While both SAP102 and PSD-95 are important for normal development of dendritic protrusions, SAP102 appears to be required for the transition from filopodia to spines. These findings of decreased presynaptic markers and prevalence of filopodia-like protrusions, suggest that SAP102 is the critical MAGUK for making initial synaptic contacts, which under normal conditions leads to synapse stabilization and spine maturation. Type-B Eph receptors (EphBs), especially EphB2 and its ligand ephrinB, have been shown to regulate spine formation and maturation as well as excitatory presynaptic and postsynaptic specialization (Dalva et al., 2000; Ethell et al., 2001; Henkemeyer et al., 2003; Penzes et al., 2003; Kayser et al.,
2006, 2008). Consequently, we tested the possibility that SAP102 and EphrinB/EphB signaling are functionally related to each other. We first examined the association between MAGUKs and EphB2 in the developing visual cortex in vivo by performing immunoprecipitations (IPs) from rat visual cortex at P14, $1 \mathrm{~d}$ after eye opening. EphB2 was coimmunoprecipitated with antiSAP102 antibody, but not with anti-PSD-95 (Fig. 4A). In agreement, SAP102, but not PSD-95, was coimmunoprecipitated with anti-EphB2 antibody from developing visual cortex (Fig. 4B). To further examine whether the association between EphB2 and SAP102 was mediated by their direct binding, we performed coimmunoprecipitation assay in HEK cells after cotransfection of EphB2 and SAP102-GFP. EphB2 was not coimmunoprecipitated with SAP102-GFP (Fig. 4C), suggesting that the association between EphB2 and SAP102 is not mediated by their direct binding, rather these two proteins are in a complex in developing cortical neurons. GluN2B, which is known to directly bind to SAP102, was coimmunoprecipitated with SAP102-GFP (Fig. 4D), showing the validity of this assay to examine direct binding.

In cortical neurons, a previous electron microscopic study showed that EphB2 is frequently detected on the surface membrane of dendritic shafts (Bouvier et al., 2008). We hypothesized that the membrane scaffold SAP102 may be required for EphB2 surface expression. Thus, we next examined whether MAGUK KD would affect surface expression of EphB2 on cortical neurons. We labeled surface proteins on DIV 14 cultured cortical neurons with sulfo-biotin, pulled down these labeled proteins with streptavidin agarose resin, and analyzed the surface protein expression by Western blotting. Proteins unbound to streptavidin resin were also collected as cytosolic fraction and analyzed. SAP102 KD caused a $24 \%$ decrease in the surface expression and a $16 \%$ increase in the cytosolic expression level of EphB2 relative to GFP control (Fig. $4 E-G)$. Neither PSD-95 KD nor PSD-93 KD changed the surface EphB2 expression level. Although there was no change in NMDAR levels at the PSD with MAGUK KDs (Fig. 2G), we examined the surface expression levels of NMDARs since the surface membrane includes both synaptic and extrasynaptic membranes. The surface expression levels of GluN1 (NR1) and GluN2A (NR2A) subunits were not significantly affected by any MAGUK KDs (Fig. 4G). SAP102 KD caused a reduction in surface GluN2B expression by $12 \%$ although it was not statistically significant. In contrast, in PSD-95 KD neurons, the surface expression level of the GluN2B (NR2B) subunit was significantly increased and the cytosolic level of GluN2B was decreased (Fig. $4 E-G)$ : a result that agrees with previous studies in which knockout or knockdown of PSD-95 showed a greater contribution of GluN2B-containing NMDAR currents (Béique et al., 2006; Elias et al., 2008). Since SAP102 can preferentially bind to the GluN2B subunit (Sans et al., 2000; Zheng et al., 2010; Chen et al., 2011), it is likely that SAP102 holds an increased amount of GluN2B at the 


\section{IP from P14 Visual Cortex}

A

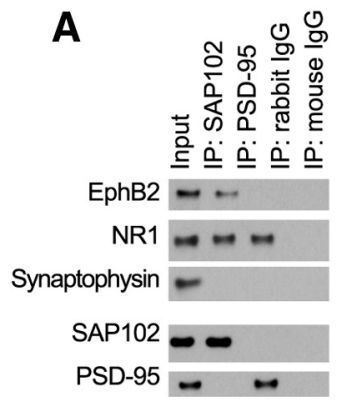

B

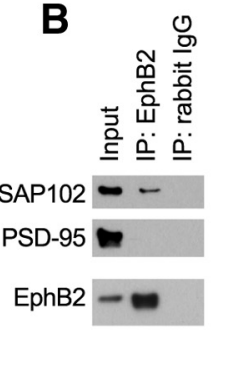

\begin{abstract}
Co-IP from HEK cells
\end{abstract}

C

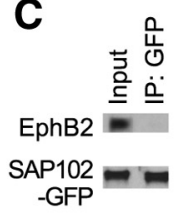

D 芌苍 GluN2B $\rightarrow \cdots$ SAP102 $\rightarrow=$

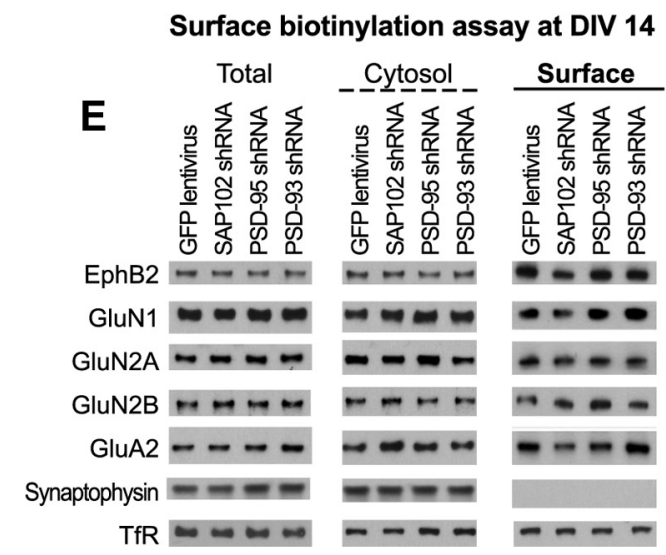

$\mathbf{F}$

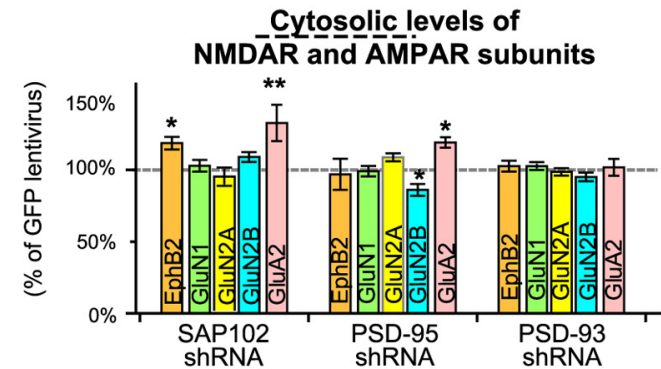

G

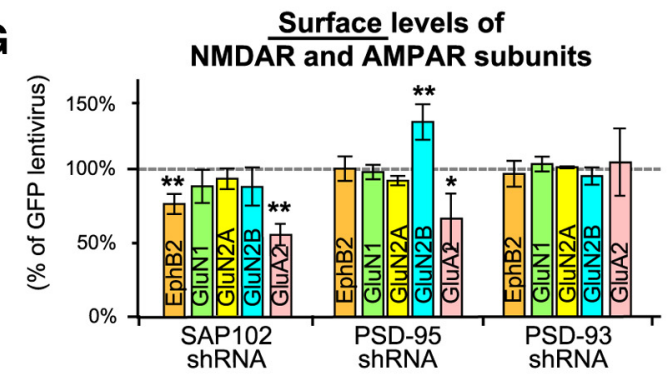

H
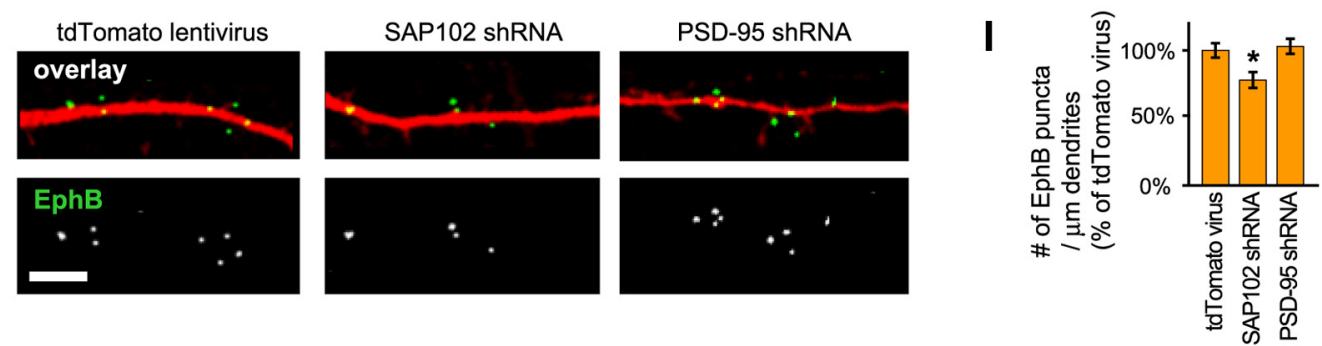

Figure 4. SAP102 is in a complex with EphB2, and SAP102 KD reduces surface expression and dendritic localization of EphB.A,B, EphB2 and SAP102 were reciprocally coimmunoprecipitated from developing (P14; 1 d after completion of eye-lid opening) rat visual cortex. IP from visual cortical homogenates showed that (A) EphB2 was immunoprecipitated with the anti-SAP102 antibody, but not with the anti-PSD-95 antibody, and that (B) SAP102, but not PSD-95, was immunoprecipitated with the anti-EphB2 antibody. Synaptophysin and PSD-95 were used as negative controls. Normal rabbit lgG and normal mouse lgG were used as additional negative controls of IP. C, D, Association between EphB2 and SAP102 is not mediated by their direct binding. C, HEK cells were cotransfected with EphB2 and SAP102-GFP expression plasmids, and coimmunoprecipitated with the anti-GFP antibody. EphB2 was not pulled down with SAP102-GFP, suggesting that these two proteins do not directly bind to each other. D, GluN2B was coimmunoprecipitated with SAP102-GFP from HEK cells cotransfected with GluN2B and SAP102-GFP, validating the reliability of coimmunoprecipitation assay in HEK cells. E-G, SAP102 shRNA reduced the surface expression level of EphB2. Cultured neurons were infected with either the GFP control, SAP102 shRNA, PSD-95 shRNA, or PSD-93 shRNA lentivirus at DIV 2. Surface proteins on DIV 14 cultured cortical neurons were biotinylated, pulled down with streptavidin agarose resin, and analyzed by Western blotting (G). The proteins unbound to streptavidin agarose resin were collected as cytosolic proteins and also analyzed $(\boldsymbol{F})$. The surface or cytosolic expression levels of EphB2, GluN1, GluN2A, GluN2B, and GluA2 for each MAGUK KD were quantified and plotted as a percentage of the GFP lentivirus control. The surface expression level of EphB2 was significantly decreased in SAP102 KD neurons. The surface expression levels of GluN1 and GluN2A were not significantly altered in any MAGUK KD neurons. However, in PSD-95 KD neurons, the surface expression level of GluN2B was increased $(\boldsymbol{G})$ and the cytosolic level of GluN2B was decreased $(\boldsymbol{F})$, suggesting that SAP102 compensates for the absence of PSD-95 by holding more GluN2B on the surface of PSD-95 KD neurons. The GluA2 AMPAR subunit was significantly decreased in the surface fraction but increased in the cytosolic fraction of SAP102 KD or PSD-95 KD neurons, suggesting both SAP102 and PSD-95 play a key role in surface AMPAR expression. Synaptophysin was used as a negative control of surface biotinylation assay. Transferrin receptor (TfR) was used as a loading control to normalize protein levels. ( $n=6$ immunoblots from 3 separate sets of cultures; data were presented as mean \pm SEM.) $H, I, S A P 102$ shRNA reduced the number of EphB puncta on dendrites. Cortical culture neurons were infected with lentiviruses expressing tdTomato only, SAP102 shRNA or PSD-95 shRNA at DIV 2, and fixed at DIV 14. EphBs were visualized by incubation with ephrinB2-Fc followed by anti-Fc and secondary antibodies (see Materials and Methods). The number of EphB puncta on dendrites was quantified and plotted as a percentage of control. These data revealed a significant decrease of EphB puncta in SAP102 KD neurons. ( $n=10$ dendrites from 5 neurons.) The density of EphB puncta per micrometer of dendrite is normalized to the tdTomato lentivirus control and presented as mean \pm SEM.

surface including extrasynaptic sites when PSD-95 is knocked down. With regard to AMPARs, SAP102 KD, and PSD-95 KD, but not PSD-93 KD, reduced surface GluA2 subunit levels (Fig. $4 E, G)$, indicating that SAP102 and PSD-95 are responsible for trafficking or retaining AMPARs on the cell surface.

Next we labeled EphBs using the ephrinB2-ligand complex (Tolias et al., 2007) (see Materials and Methods) in tdTomatolentivirus control, SAP102 KD, and PSD-95 KD neurons at DIV 14. The density of EphB puncta on dendrites was decreased by $22 \%$ in SAP102 KD neurons compared to tdTomato lentivirus infected or PSD-95 KD neurons (Fig. 4H,I). Together, these results show that SAP102 KD reduces surface and dendritic expression of EphB in cultured young cortical neurons.

SAP102 KD impairs ephrinB/EphB-induced actin reorganization, synapse formation, and synaptic AMPAR trafficking

Next we investigated whether SAP102 KD affects EphB function. Known roles of ephrinB/EphB signaling include actin reorganization, synapse formation, and AMPAR trafficking. 
Upon stimulation with ephrinB ligand, EphB activates its downstream signaling cascade consisting of Kalirin-7, Rac1, and group I PAKs (PAK1-3); these in turn regulate actin cytoskeleton remodeling (Penzes et al., 2003). We first examined ephrinB/EphB-induced actin reorganization. The ligand ephrinB2 works as a dimer; thus, to activate EphB signaling, we used ephrinB2 fused with the Fc portion of Ig molecule, which allows ephrinB2 to be dimerized with an anti-Fc antibody (Dalva et al., 2000). We treated cultured cortical neurons with dimerized ephrinB2-Fc for $1 \mathrm{~h}$. As a control, we treated neurons with dimerized Fc alone. which without ephrinB attached would not be expected to activate EphB. We visualized filamentous actin (F-actin) using phalloidin conjugated with fluorescent dye. We quantified the signal intensity of phalloidin within GFP-positive, lentivirus-infected dendrites and dendritic protrusions. In the control (Fc-treated) condition, there was no significant difference in phalloidin signal intensity between GFP lentivirus, SAP102 shRNA1, SAP102 shRNA2, PSD-95 shRNA1, and PSD-95 shRNA2 neurons. However, after $1 \mathrm{~h}$ treatment with ephrinB2-Fc, phalloidin signal intensity showed a robust increase especially in dendritic protrusions of GFP lentivirus as well as PSD-95 KD neurons. In contrast, in SAP102 KD neurons, there was no increase in phalloidin signal intensity after EphB activation suggesting ephrinB/EphBinduced reorganization of actin filaments is abolished in SAP102 KD neurons (Fig. $5 A, B$ ).

Next we analyzed whether SAP102 KD affects ephrinBinduced presynaptic formation since ephrinB treatment has been shown to increase the density of presynaptic markers (Dalva et al., 2000; Penzes et al., 2003). DIV 14 cortical neurons were treated with ephrinB2-Fc or control Fc, and immunostained for the presynaptic marker Bassoon. In the control (Fc-treated) condition, SAP102 KD and PSD-95 KD neurons have a significantly fewer number of Bassoon puncta on dendrites than GFP lentivirus neurons, as seen in Figure $2 \mathrm{~A}$. After ephrinB2-Fc treatment, the density of Bassoon puncta associated with lentivirus-infected dendrites and dendritic protrusions was significantly increased in GFP lentivirus and PSD-95 KD neurons. However, this ephrinB/ EphB-dependent increase in Bassoon puncta was not observed in SAP102 KD neurons (Fig. 5C,D).

We also examined whether SAP102 KD impairs ephrinB/ EphB-induced AMPAR trafficking since EphB2 activation has been shown to increase AMPAR on the cell surface (Kayser et al., 2006). We treated cultured cortical neurons with dimerized ephrinB2-Fc or control Fc, and then prepared PSD fractions from GFP lentivirus, SAP102 KD, and PSD-95 KD neurons at DIV 14. In the control (Fc-treated) condition, SAP102 KD and PSD-95 KD neurons showed 48 and $31 \%$ reductions in the amount of the GluA2 AMPAR subunit in the PSD fraction, as seen in Figure 2, E and $F$. EphB activation with ephrinB significantly increased the level of GluA2 subunits in the PSD fraction of GFP lentivirus as well as PSD-95 KD neurons, but not in SAP102 KD neurons (Fig. $5 E, F)$, suggesting that SAP102 is necessary for ephrinB/EphBinduced AMPAR trafficking. The whole lysate expression levels of AMPAR and NMDAR receptor subunits were not significantly altered with ephrinB-Fc treatment (data not shown).

\section{Kalirin-7, SAP102, and GluN2B form a protein complex in developing visual cortex}

The results above suggest that EphB signaling is functionally impaired in SAP102 KD neurons. Next we investigated the interaction between MAGUKs and the signaling molecules that can modulate ephrinB/EphB function. Kalirin-7 is a neuronal Rac1-
GEF and characterized as a downstream mediator of the EphB signaling pathway (Penzes and Jones, 2008; Ma, 2010). After EphB activation with ephrinB-Fc, Kalirin-7 is recruited to synapses for subsequent activation of Racl and PAK kinase, which then promote spine morphogenesis (Penzes et al., 2003). Kalirin-7 is also necessary for neuronal activity-dependent AMPAR trafficking and spine maturation (Xie et al., 2007). Kalirin-7 has a PDZ binding motif at its $C$ terminus and can bind to PDZ domain containing proteins including MAGUKs (Penzes et al., 2001). To determine whether MAGUKs interact with Kalirin-7 in the neonatal visual cortex, we performed IP from developing rat visual cortex at P14 after eye-lid opening with an anti-SAP102 or anti-PSD-95 antibody. Anti-pan-Kalirin antibody recognizes several Kalirin isoforms in Western blotting as previously reported, and Kalirin-7 appears around $190 \mathrm{kDa}$ while other isoforms, including Kalirin-9 and Kalirin-12, appear in the higher molecular weight regions (Penzes et al., 2000; Nolt et al., 2011). Among several Kalirin isoforms, Kalirin-7 was specifically pulled down with the anti-SAP102 antibody from young visual cortex, but not with the anti-PSD-95 antibody (Fig. 6A). We also examined interactions between MAGUKs and NMDAR subunits in the intact developing visual cortex. Both SAP102 and PSD-95 pulled down similar amounts of the GluN1 subunit. However, SAP102 pulled down more GluN2B subunits than PSD-95, while PSD-95 pulled down more GluN2A subunits than SAP102 (Fig. 6A). These results confirmed the preferential interaction between SAP102 and GluN2B, and between PSD-95 and GluN2A in the early visual cortex as previously shown in the hippocampus (Sans et al., 2000).

In adult visual cortex, Karilin-7 was pulled down by both SAP102 and PSD-95 (Fig. 6B). The difference between neonatal and adult cortex is probably due to developmental expression pattern of MAGUKs and NMDAR subunits. In the early postnatal brain, SAP102 is highly expressed and present at synapses, whereas PSD-95 expression does not reach its highest peak and continues to increase until adult. (Yoshii et al., 2003). GluN2B is also highly expressed in the neonatal cortex (van Zundert et al., 2004), binds directly to Kalirin-7 (Kiraly et al., 2011), and preferentially binds to SAP102 especially in early cortex (Fig. 6A). Therefore, it is likely that Kalirin-7 and SAP102 are in a stable complex with GluN2B in the early brain to regulate cortex development (see Discussion).

\section{PAK kinase activity is downregulated by SAP102 KD}

Group 1 PAKs, including PAK1, PAK2, and PAK3, are the key downstream components of EphB signaling (Penzes et al., 2003; Kayser et al., 2008); one of their main functions is regulation of the actin cytoskeleton (Kreis and Barnier, 2009). Importantly, mutations in PAK3, like SAP102, are found in human XLMR patients (Allen et al., 1998; Bienvenu et al., 2000; Rejeb et al., 2008).

To analyze the activity level of Group 1 PAKs, we used antiPAK phospho-serine 144/141/139 antibody, which detects essential phosphorylation sites for the activation and maintenance of their kinase activity (Kreis and Barnier, 2009). DIV 14 cortical neurons were treated with ephrinB2-Fc or control Fc, and homogenates were analyzed by immunoblotting. Under basal (Fctreated) conditions, SAP102 KD neurons exhibited a $40 \%$ decrease in PAK phosphorylation level relative to GFP lentivirus and PSD-95 KD neurons (Fig. 7A,B). Activation of EphB with ephrinB increased the PAK phosphorylation level in GFP lentivirus control and PSD-95 KD neurons by 50\%, but failed to increase the PAK phosphorylation level in SAP102 KD neurons, suggesting that SAP102 is required for PAK activity. Neither SAP102 KD nor PSD-95 KD altered the total expression levels of 
A

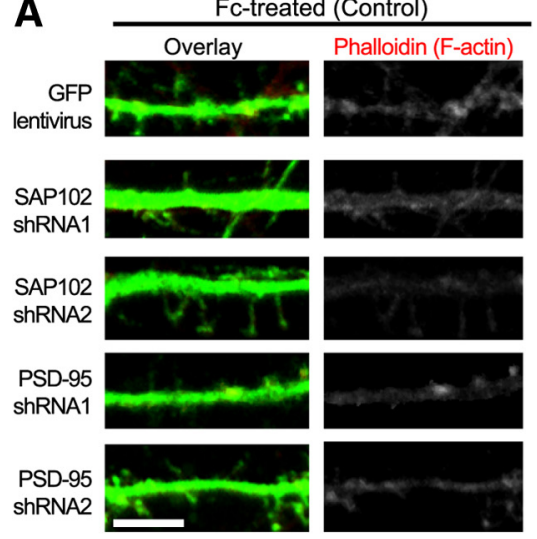

C

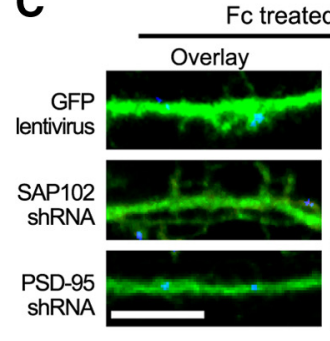

Fc treated (Control)
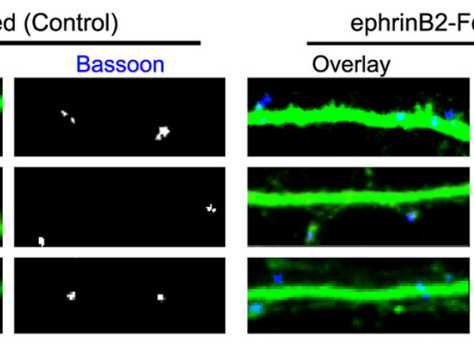

$\mathbf{E}$

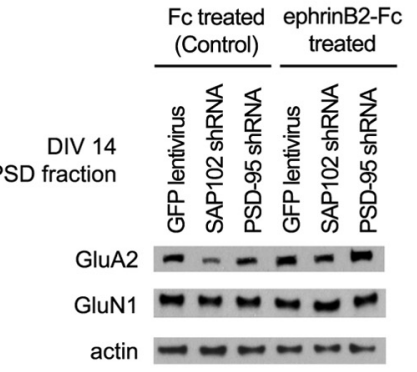

ephrinB2-Fc-treated

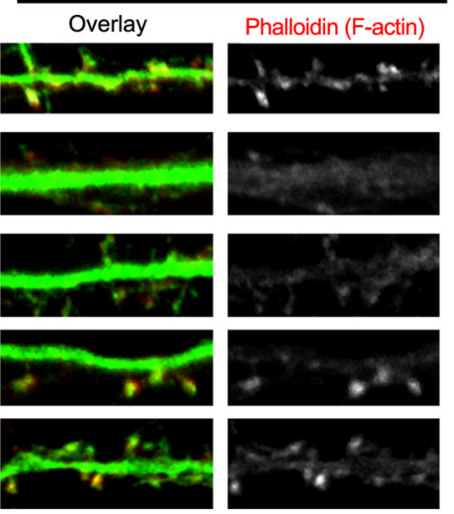

B

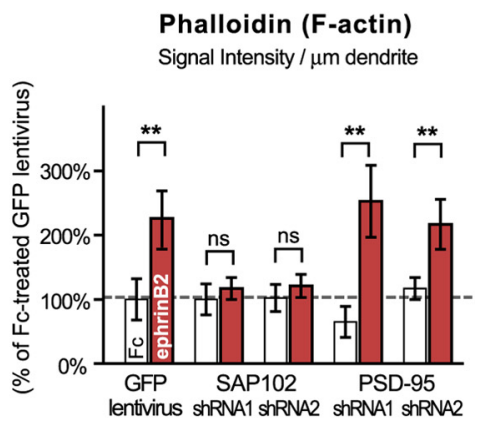

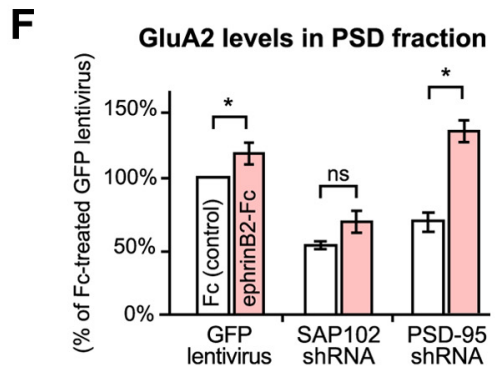

Figure 5. SAP102 shRNAs impaired ephrinB-induced actin reorganization, synapse formation, and AMPAR trafficking. $A, B$, SAP102 shRNA impaired ephrinB-induced reorganization of actin filaments. DIV 14 cortical neuron cultures were treated with dimerized Fc (control) or ephrinB2-Fc for $1 \mathrm{~h}$, and stained with phalloidin conjugated with Alexa 543 to visualize the F-actin. In the Fc-treated (control) conditions, phalloidin signals within GFP-positive lentivirus-infected dendrites and dendritic protrusions were comparable among neurons infected with lentivirus expressing GFP only, SAP102 shRNA1, SAP102 shRNA2, PSD-95 shRNA1, and PSD-95 shRNA2. After ephrinB2-Fc treatment, GFP lentivirus as well as PSD-95 KD neurons showed > 2-fold increases in phalloidin signal intensity. However, ephrinB treatment failed to increase phalloidin signal in both SAP102 shRNA1 and SAP102 shRNA2 neurons. The phalloidin signal intensity per micrometer of dendrite is normalized to Fc-treated GFP lentivirus neurons and presented as mean $0 \pm$ SEM ( $n=>10$ dendrites from 5 neurons). C, D, SAP102 KD impaired ephrinB-induced synapse formation. DIV 14 cortical neurons were treated with dimerized $\mathrm{Fc}$ (control) or ephrinB2-Fc for $1 \mathrm{~h}$, and immunostained for the presynaptic marker Bassoon. EphrinB2-Fc treatment significantly increased Bassoon puncta density by $>30 \%$ in GFP lentivirus neurons as well as PSD-95 KD neurons, but did not cause a significant increase in SAP102 KD neurons. Bassoon puncta associated with GFP-positive lentivirusinfected dendrites and dendritic protrusions were analyzed, and the density of Bassoon puncta is normalized to Fc-treated GFP lentivirus-infected neurons and presented as mean \pm SEM $(n=>10$ dendrites from 5 neurons). E, F, SAP102 KD impaired ephrinB-induced synaptic AMPAR trafficking. DIV 14 cortical culture neurons were treated with dimerized Fc (control) or ephrinB2-Fc for 1 h, and PSD fractions were prepared to analyze the amount of AMPAR GluA2 subunit and of NMDAR GluN1subunit by Western blotting. In Fc-treated (control) conditions, the amounts of GluA2 subunit in PSD fractions were significantly lower in both SAP102 KD and PSD-95 KD neurons compared with GFP lentivirus-infected neurons. EphrinB2-Fc treatment increased the amounts of GluA2 subunit in PSD fractions of the GFP lentivirus as well as the PSD-95 KD neurons. However, in SAP102 KD neurons, this EphrinB2-Fc-induced increase in synaptic AMPARs was abolished. The levels of GluA2 in the PSD fraction is normalized to Fc-treated GFP lentivirus infected neurons and presented as mean \pm SEM ( $n=3$ independently prepared and analyzed sets of cultures).

PAK1 and PAK3, both of which are the abundant group I PAKs in neurons (Kreis and Barnier, 2009). These results revealed functional association between two mental retardation genes, SAP102 and PAK3, and suggest that the downregulation of PAK kinase activity underlies the impaired synapse development phenotype observed in SAP102 KD neurons.

\section{Discussion}

This work was motivated by previous intensive studies of the rodent visual pathway before and after eye opening where we have been able to follow rapid in vivo changes by manipulating the onset of pattern vision (Yoshii et al., 2003, 2011; Lu and Constantine-Paton, 2004; Phillips et al., 2011). The move to cul- tured neurons for this study allowed us to examine specific properties of the SAP102 MAGUK under controlled conditions. The few experiments presented here used brain tissue from the visual cortex after eye opening because of our familiarity with the developmental state of the intact system at this time.

SAP102 and PSD-95 are the major MAGUKs regulating excitatory synaptogenesis and synaptic AMPAR levels in developing cortical neurons

The current studies based on quantitative immunocytochemistry and immunoblotting demonstrate that knockdowns of SAP102 and PSD-95, but not PSD-93, decrease excitatory synaptogenesis and both synaptic and surface levels of AMPARs. The results 
A

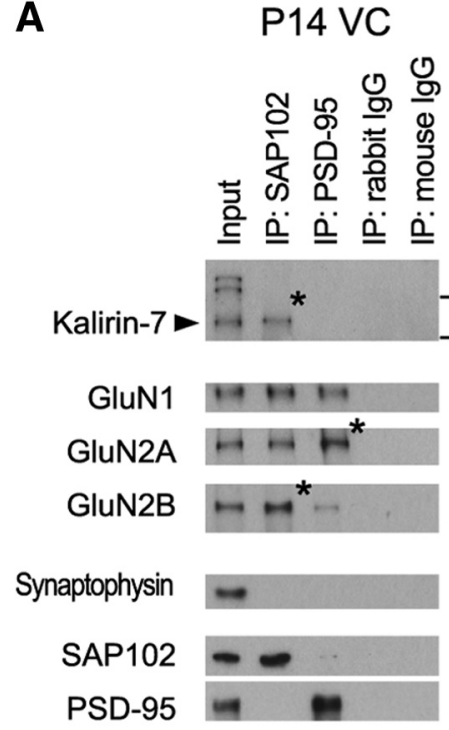

B
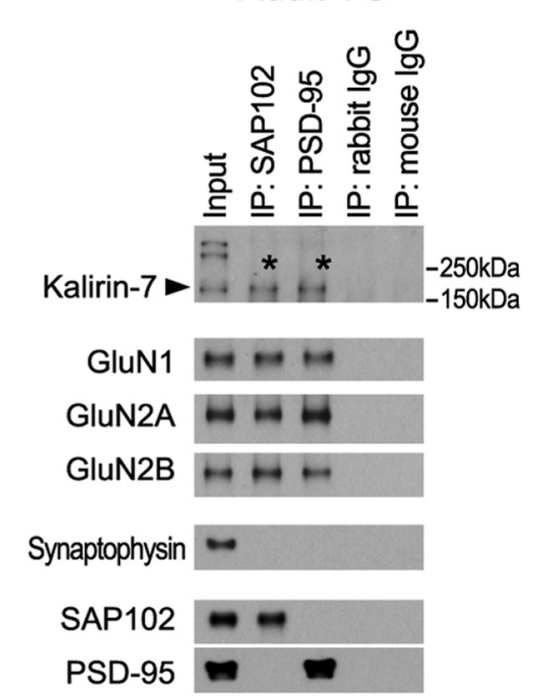

Figure 6. Rac1-GEF Kalirin-7 and the GluN2B NMDAR subunit are preferentially immunoprecipitated with SAP102 from the developing rat visual cortex. A, Proteins immunoprecipitated with an anti-SAP102 or an anti-PSD-95 antibody from P14 visual cortex were analyzed by Western blotting. Anti-pan Kalirin antibody detects several isoforms: Kalirin-7 around 190 kDa and Kalirin- 9 and Kalirin- 12 in the higher molecular weight region. Among Kalirin isoforms, only Kalirin-7 was preferentially pulled down with SAP102 as indicated by an asterisk. Both SAP102 and PSD-95 pulled down a similar amount of the GluN1 NMDAR subunit. However, SAP102 pulled down more GluN2B subunit than PSD-95, in contrast, PSD-95 pulled down higher levels of the GluN2A subunit than SAP102, showing preferential interactions between SAP102 and GluN2B and between PSD-95 and GluN2A in intact developing visual cortex. Asterisks indicate Kalirin-7 at 190 kDa pulled down with SAP102, GluN2A pulled down PSD-95, and GluN2B pulled down with SAP102. Synaptophysin, a presynaptic vesicle associated protein, was shown as a negative control. Normal rabbit lgG and normal mouse lgG were used as negative controls of IP. B, Proteins immunoprecipitated from adult visual cortex were analyzed by Western blotting. Kalirin-7 was pulled down by both anti-SAP102 and anti-PSD-95 antibodies showing a difference in protein interaction between developing and mature visual cortex.

A Fc treated ephrinB2-Fc B
(Control) treated

DIV 14 Whole Lysate
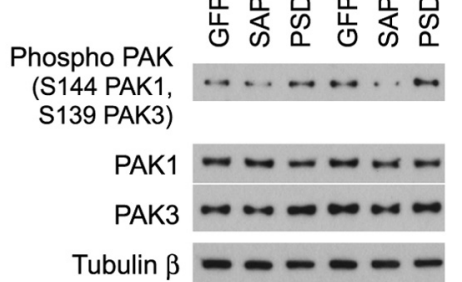

\section{B}

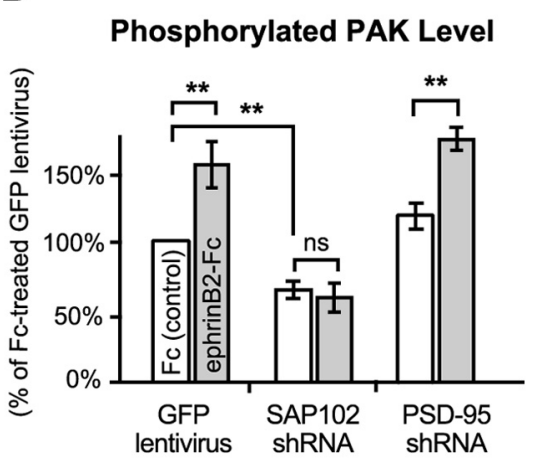

Figure 7. SAP102 shRNA downregulates group I PAK activity. $A, B$, Group I PAK activity was analyzed with an anti-phospho PAK antibody recognizing phosphorylated serines at 144 of PAK1, 141 of PAK2, and 139 of PAK3; these phosphorylations are necessary for activation and maintenance of PAK activity. DIV 14 cultured cortical neurons were treated with dimerized control Fc or ephrinB2-Fc for $1 \mathrm{~h}$, and whole lysates were prepared and analyzed by Western blotting. Under the control condition (Fc-treated), SAP102 KD neurons showed a significantly reduced level of PAK phosphorylation compared with GFP lentivirus or PSD-95 KD neurons. After a $1 \mathrm{~h}$ treatment with ephrinB2-Fc, the PAK phosphorylation level was increased by $>50 \%$ in GFP lentivirus as well as PSD-95 KD neurons. However, in SAP102 KD neurons, the PAK phosphorylation levels remained unchanged and were significantly lower than GFP lentivirus or PSD-95 KD neurons, suggesting PAK activity is downregulated in SAP102 KD neurons. There were no significant differences in the expression levels of either PAK1 or PAK3 between GFP lentivirus, SAP102 KD, and PSD-95 KD neurons. ( $n=3$ independently prepared and analyzed sets of cultures, data is normalized to Fc-treated GFP lentivirus infected neurons and presented as mean \pm SEM.)

indicate that SAP102 and PSD-95 are critical MAGUKs in young cortical neurons: a difference from conclusions reached with older hippocampal neurons where PSD-95 and PSD-93 control synaptic plasticity (Elias et al., 2006, 2008). This difference could be due to the relative abundance and/or developmental expression patterns of
MAGUKs. During cortical development, PSD-93 expression increases later than SAP102 and PSD-95 (Fig. $1 A, B$ ), and knockdown of PSD-93 does not significantly affect the total MAGUK level in young cortical neurons (Fig. 1D,E). The fourth synaptic MAGUK family member, SAP97, is not prevalent in young cortical neurons (Fig. 1D,E). This is consistent with a previous quantitative proteomic study (Cheng et al., 2006).

\section{Evidence for a SAP102, GluN2B- NMDARs, Kalirin-7, and EphB2 complex in early cortex}

The most notable knockdown phenotype observed in the present study is the increased length of dendritic protrusions caused by SAP102 shRNAs, suggesting that SAP102 is normally involved in regulation of the actin cytoskeleton. The density of dendritic protrusions was not significantly altered by SAP102 shRNAs (Fig. 3B). This is consistent with a previous study conducted in hippocampal slices (Elias et al., 2008). Another study showed that a particular splice variant of SAP102 containing the L1 region plays a role in spine morphology. Specific knockdown of the L1 regioncontaining SAP102, which does not affect other SAP102 splice variants, resulted in shortening of dendritic protrusions (Chen et al., 2011). However, mutations in human mental retardation patients cause the loss of total SAP102 expression; thus, we consequently knocked down all SAP102 splice variants and observed an increase in the length of dendritic protrusions. It is therefore interesting to see a difference in the effects on dendritic protrusion morphology between knockdown of the L1 splice variant alone (Chen et al., 2011) and knockdown of all splice variants (Fig. 3). Knockdown of all splice variants appears to mask the effect of knockdown of the L1 splice variant alone, suggesting different, potentially opposing, functions for the L1 and at least one of the other splice variants of SAP102.

We subsequently studied the relationship between SAP102 and EphB/Kalirin7/PAK signaling. Kalirin-7 interacts with MAGUKs via their PDZ-binding motif (Penzes et al., 2001), but its interaction with SAP102 in early development had not been addressed. Our study reveals that Kalirin-7 selectively associates with SAP102 in developing visual cortex and that SAP102 binds relatively more of the GluN2B than the GluN2A NMDAR subunit (Fig. 6A). In adult visual cortex, Kalirin-7 associates with both SAP102 and PSD-95 as previously shown in hippocampus (Penzes et al., 2001). One possible explanation for this difference is that in the developing cortex SAP102 
is the predominant postsynaptic scaffold because PSD-95 is not yet fully expressed. Another possible explanation is that SAP102 and Kalirin-7 are in a stable protein complex in the early brain because of GluN2B. A recent study showed that the L1 SAP102 splice variant binds specifically to the cytoplasmic tail of GluN2B (Chen et al., 2011). Another study showed direct binding of Kalirin-7 to the juxtamembrane cytoplasmic region of GluN2B, but not GluN2A (Kiraly et al., 2011). Here we show that SAP102 and EphB2 reciprocally immunoprecipitate each other from young visual cortex (Fig. 4A,B). SAP102 associated with GluN2B is found on dendritic shafts in young neurons (Washbourne et al., 2004), and EphB2 is also expressed along dendritic shafts as well as at postsynaptic sites in cortical neurons (Bouvier et al., 2008). Since EphB2 binds to NMDARs (Dalva et al., 2000), it is likely that all four molecules in this pathway are present in the same complex and organized at synapses by SAP102. Such a complex may serve to control early NMDAR functions since EphB activation regulates surface GluN2B expression (Nolt et al., 2011) and phosphorylates GluN2B, thereby increasing calcium influx through NMDARs (Takasu et al., 2002). Thus these results suggest that SAP102 mediates critical cross talk between NMDAR function and $\mathrm{EphB}$ signaling to facilitate normal cortical synapse formation and maturation.

\section{Rac/Rho/cdc42 GEFs and GTPase activating proteins in brain development}

This study reveals that knockdown of SAP102 impairs downstream functions of ephrinB/EphB signaling including actin reorganization, synaptogenesis, and AMPAR trafficking. Given the diverse functions of Kalirin-7 including spine morphogenesis, excitatory synaptogenesis and synaptic plasticity (Ma et al., 2003, 2008; Penzes et al., 2003; Xie et al., 2007), association between Karilin-7 and the SAP102-NMDAR complex is likely to be critical to developmental synaptic plasticity.

In addition to Kalirin-7, several other Rho/Rac/cdc42 GEFs, including Tiam 1 , Intersectin, $\alpha$ PIX, and $\beta$ PIX, are present in dendrites or spines. Tiam 1 and Intersectin have been shown to interact with EphB2 and mediate spine and synapse formation (Irie and Yamaguchi, 2002; Tolias et al., 2007). $\alpha$ PIX, another causal gene for XLMR, and $\beta$ PIX have been shown to interact with Shank, GIT1, and PAK, and regulate spine morphology and synapse formation (Zhang et al., 2005; Nodé-Langlois et al., 2006; Saneyoshi et al., 2008). We also examined the interactions between MAGUKs and $\alpha$ PIX or $\beta$ PIX, but we could find no evidence for a definitive interaction between these molecules in the neonatal cortex. In this context, it is important to note that several additional Rho/Rac/cdc42 GTPase activating proteins, such as Oligopherenin (Nadif Kasri et al., 2009), BCR, and ABR (Oh et al., 2010), are found in dendrites and spines so that there are multiple mechanisms by which neurons control Rac activity and modulate actin dynamics during dendritic spine growth and synapse formation.

\section{PAK is a downstream key player in SAP102 scaffolded signaling and cause of mental retardation}

This study also demonstrates that knockdown of SAP102 suppresses the activity of group I PAKs that are downstream effectors of EphB and Kalirin-7. It is therefore important to note that elongated dendritic filopodia are increased after knockdown of PAK3 or overexpression of the mutated PAK3 found in human mental retardation patients (Boda et al., 2004). Consequently, perturbation of group I PAK functions is likely responsible for the protrusion abnormalities observed in SAP102 KD neurons.
Involvement of SAP102 and PAK3 in cognitive function was previously shown in SAP102 KO (Cuthbert et al., 2007) and PAK3 KO mice (Meng et al., 2005), both of which exhibit deficits in learning and memory. Here we demonstrate the functional association between two mental retardation genes: SAP102 and PAK3, and the unique contribution of SAP102 to early cortical development. It is likely that the disruption of the PAK signaling cascade we demonstrate here is responsible for impaired synapse formation and cognitive deficits in patients with XLMR caused by SAP102 mutations.

\section{Conclusion}

NMDA and AMPA glutamate receptors and their associated PSD proteins serve critical roles in the activity-dependent development and plasticity of spines and synapses. However, the MAGUK family scaffolds organize signaling complex of these two major excitatory glutamate receptors, and SAP102 is the earliest appearing postsynaptic scaffold. The importance of the PSD molecules for the normal brain development has been demonstrated by the facts that many PSD proteins, including Shank, Neuroligin, and Neurexin, are associated with neurodevelopmental disorders such as autism and mental retardation (Ropers, 2010; Scherer and Dawson, 2011). However, little is known about which PSD proteins are preferentially associated with SAP102, when and where these protein complexes are formed, and how these interactions modulate synapse maturation in the developing brain. Here we show that SAP102 is necessary for EphB/PAK signaling and proper glutamate synapse development. Thus it will be interesting to further study how neurons use the potentially large number of other signaling molecules coordinated by SAP102 to facilitate the development of normal brain circuitry. Uncovering the developmental functions of dozens of these molecules is a formidable challenge, but it will be necessary to study them to provide deeper understandings of early brain development and disease etiology. There is broad agreement that identifying single synaptic molecules found to be defective in human patients is the first step in finding cures for devastating neurological diseases. However, particularly for childhood onset disease, identifying the complexes these molecules form and their developmental appearance is a critical next step in using this genetic information to instruct early intervention and drug development for the potential treatment of lifelong neurological dysfunction that most neurodevelopmental disorders impose.

\section{References}

Allen KM, Gleeson JG, Bagrodia S, Partington MW, MacMillan JC, Cerione RA, Mulley JC, Walsh CA (1998) PAK3 mutation in nonsyndromic X-linked mental retardation. Nat Genet 20:25-30. CrossRef Medline

Banker G, Goslin K (1991) Culturing nerve cells. Cambridge, MA: MIT.

Béique JC, Lin D-T, Kang MG, Aizawa H, Takamiya K, Huganir RL (2006) Synapse-specific regulation of AMPA receptor function by PSD-95. Proc Natl Acad Sci U S A 103:19535-19540. CrossRef Medline

Bienvenu T, des Portes V, McDonell N, Carrié A, Zemni R, Couvert P, Ropers $\mathrm{HH}$, Moraine C, van Bokhoven H, Fryns JP, Allen K, Walsh CA, Boué J, Kahn A, Chelly J, Beldjord C (2000) Missense mutation in PAK3, R67C, causes X-linked nonspecific mental retardation. Am J Med Genet 93:294298. CrossRef Medline

Boda B, Alberi S, Nikonenko I, Node-Langlois R, Jourdain P, Moosmayer M, Parisi-Jourdain L, Muller D (2004) The mental retardation protein PAK3 contributes to synapse formation and plasticity in hippocampus. J Neurosci 24:10816-10825. CrossRef Medline

Boda B, Dubos A, Muller D (2010) Signaling mechanisms regulating synapse formation and function in mental retardation. Curr Opin Neurobiol 20:519-527. CrossRef Medline

Bouvier D, Corera AT, Tremblay ME, Riad M, Chagnon M, Murai KK, Pasquale EB, Fon EA, Doucet G (2008) Presynaptic and post-synaptic lo- 
calization of EphA4 and EphB2 in adult mouse forebrain. J Neurochem 106:682-695. CrossRef Medline

Chen BS, Thomas EV, Sanz-Clemente A, Roche KW (2011) NMDA receptor-dependent regulation of dendritic spine morphology by SAP 102 splice variants. J Neurosci 31:89-96. CrossRef Medline

Cheng D, Hoogenraad CC, Rush J, Ramm E, Schlager MA, Duong DM, Xu P, Wijayawardana SR, Hanfelt J, Nakagawa T, Sheng M, Peng J (2006) Relative and absolute quantification of postsynaptic density proteome isolated from rat forebrain and cerebellum. Mol Cell Proteomics 5:11581170. CrossRef Medline

Cuthbert PC, Stanford LE, Coba MP, Ainge JA, Fink AE, Opazo P, Delgado JY, Komiyama NH, O’Dell TJ, Grant SG (2007) Synapse-associated protein 102/dlgh3 couples the NMDA receptor to specific plasticity pathways and learning strategies. J Neurosci 27:2673-2682. CrossRef Medline

Dalva MB, Takasu MA, Lin MZ, Shamah SM, Hu L, Gale NW, Greenberg ME (2000) EphB receptors interact with NMDA receptors and regulate excitatory synapse formation. Cell 103:945-956. CrossRef Medline

Dittgen T, Nimmerjahn A, Komai S, Licznerski P, Waters J, Margrie TW, Helmchen F, Denk W, Brecht M, Osten P (2004) Lentivirus-based genetic manipulations of cortical neurons and their optical and electrophysiological monitoring in vivo. Proc Natl Acad Sci U S A 101:18206-18211. CrossRef Medline

Ehlers MD (2003) Activity level controls postsynaptic composition and signaling via the ubiquitin-proteasome system. Nat Neurosci 6:231-242. CrossRef Medline

Ehrlich I, Klein M, Rumpel S, Malinow R (2007) PSD-95 is required for activity-driven synapse stabilization. Proc Natl Acad Sci U S A 104:41764181. CrossRef Medline

El-Husseini AE, Schnell E, Chetkovich DM, Nicoll RA, Bredt DS (2000) PSD-95 involvement in maturation of excitatory synapses. Science 290: 1364-1368. Medline

Elias GM, Nicoll RA (2007) Synaptic trafficking of glutamate receptors by MAGUK scaffolding proteins. Trends Cell Biol 17:343-352. CrossRef Medline

Elias GM, Funke L, Stein V, Grant SG, Bredt DS, Nicoll RA (2006) Synapsespecific and developmentally regulated targeting of AMPA receptors by a family of MAGUK scaffolding proteins. Neuron 52:307-320. CrossRef Medline

Elias GM, Elias LA, Apostolides PF, Kriegstein AR, Nicoll RA (2008) Differential trafficking of AMPA and NMDA receptors by SAP102 and PSD-95 underlies synapse development. Proc Natl Acad Sci U S A 105:2095320958. CrossRef Medline

Ethell IM, Irie F, Kalo MS, Couchman JR, Pasquale EB, Yamaguchi Y (2001) EphB/syndecan-2 signaling in dendritic spine morphogenesis. Neuron 31:1001-1013. CrossRef Medline

Funke L, Dakoji S, Bredt DS (2005) Membrane-associated guanylate kinases regulate adhesion and plasticity at cell junctions. Annu Rev Biochem 74:219-245. CrossRef Medline

Futai K, Kim MJ, Hashikawa T, Scheiffele P, Sheng M, Hayashi Y (2007) Retrograde modulation of presynaptic release probability through signaling mediated by PSD-95-neuroligin. Nat Neurosci 10:186-195. CrossRef Medline

Gerrow K, Romorini S, Nabi SM, Colicos MA, Sala C, El-Husseini A (2006) A performed complex of postsynaptic proteins is involved in excitatory synapse development. Neuron 49:547-562. CrossRef Medline

Gray JA, Shi Y, Usui H, During MJ, Sakimura K, Nicoll RA (2011) Distinct modes of AMPA receptor suppression at developing synapses by GluN2A and GluN2B: single-cell NMDA receptor subunit deletion in vivo. Neuron 71:1085-1101. CrossRef Medline

Henkemeyer M, Itkis OS, Ngo M, Hickmott PW, Ethell IM (2003) Multiple EphB receptor tyrosine kinases shape dendritic spines in the hippocampus. J Cell Biol 163:1313-1326. CrossRef Medline

Humeau Y, Gambino F, Chelly J, Vitale N (2009) X-linked mental retardation: focus on synaptic function and plasticity. J Neurochem 109:1-14. CrossRef Medline

Irie F, Yamaguchi Y (2002) EphB receptors regulate dendritic spine development via intersectin, Cdc42 and N-WASP. Nat Neurosci 5:1117-1118. CrossRef Medline

Kayser MS, McClelland AC, Hughes EG, Dalva MB (2006) Intracellular and trans-synaptic regulation of glutamatergic synaptogenesis by EphB receptors. J Neurosci 26:12152-12164. CrossRef Medline

Kayser MS, Nolt MJ, Dalva MB (2008) EphB receptors couple dendritic filopodia motility to synapse formation. Neuron 59:56-69. CrossRef Medline

Kiraly DD, Lemtiri-Chlieh F, Levine ES, Mains RE, Eipper BA (2011) Kalirin binds the NR2B subunit of the NMDA receptor, altering its synaptic localization and function. J Neurosci 31:12554-12565. CrossRef Medline

Klein R (2009) Bidirectional modulation of synaptic functions by Eph/ephrin signaling. Nat Neurosci 12:15-20. CrossRef Medline

Kreis P, Barnier JV (2009) PAK signalling in neuronal physiology. Cell Signal 21:384-393. CrossRef Medline

Lai KO, Ip NY (2009) Synapse development and plasticity: roles of ephrin/ Eph receptor signaling. Curr Opin Neurobiol 19:275-283. CrossRef Medline

Lois C, Hong EJ, Pease S, Brown EJ, Baltimore D (2002) Germline transmission and tissue-specific expression of transgenes delivered by lentiviral vectors. Science 295:868-872. CrossRef Medline

Lu W, Constantine-Paton M (2004) Eye opening rapidly induces synaptic potentiation and refinement. Neuron 43:237-249. CrossRef Medline

Ma XM (2010) Kalirin-7 is a key player in the formation of excitatory synapses in hippocampal neurons. ScientificWorldJournal 10:1655-1666. CrossRef Medline

Ma XM, Huang J, Wang Y, Eipper BA, Mains RE (2003) Kalirin, a multifunctional Rho guanine nucleotide exchange factor, is necessary for maintenance of hippocampal pyramidal neuron dendrites and dendritic spines. J Neurosci 23:10593-10603. Medline

Ma XM, Kiraly DD, Gaier ED, Wang Y, Kim EJ, Levine ES, Eipper BA, Mains RE (2008) Kalirin-7 is required for synaptic structure and function. J Neurosci 28:12368-12382. CrossRef Medline

Meng J, Meng Y, Hanna A, Janus C, Jia Z (2005) Abnormal long-lasting synaptic plasticity and cognition in mice lacking the mental retardation gene Pak3. J Neurosci 25:6641-6650. CrossRef Medline

Nadif Kasri N, Nakano-Kobayashi A, Malinow R, Li B, Van Aelst L (2009) The Rho-linked mental retardation protein oligophrenin-1 controls synapse maturation and plasticity by stabilizing AMPA receptors. Genes Dev 23:1289-1302. CrossRef Medline

Nakagawa T, Futai K, Lashuel HA, Lo I, Okamoto K, Walz T, Hayashi Y, Sheng M (2004) Quaternary structure, protein dynamics, and synaptic function of SAP97 controlled by L27 domain interactions. Neuron 44: 453-467. CrossRef Medline

Nodé-Langlois R, Muller D, Boda B (2006) Sequential implication of the mental retardation proteins ARHGEF6 and PAK3 in spine morphogenesis. J Cell Sci 119:4986-4993. CrossRef Medline

Nolt MJ, Lin Y, Hruska M, Murphy J, Sheffler-Colins SI, Kayser MS, Passer J, Bennett MV, Zukin RS, Dalva MB (2011) EphB controls NMDA receptor function and synaptic targeting in a subunit-specific manner. J Neurosci 31:5353-5364. CrossRef Medline

Oh D, Han S, Seo J, Lee JR, Choi J, Groffen J, Kim K, Cho YS, Choi HS, Shin H, Woo J, Won H, Park SK, Kim SY, Jo J, Whitcomb DJ, Cho K, Kim H, Bae YC, Heisterkamp N, et al. (2010) Regulation of synaptic Rac1 activity, long-term potentiation maintenance, and learning and memory by BCR and ABR Rac GTPase-activating proteins. J Neurosci 30:1413414144. CrossRef Medline

Penzes P, Jones KA (2008) Dendritic spine dynamics-a key role for kalirin-7. Trends Neurosci 31:419-427. CrossRef Medline

Penzes P, Johnson RC, Alam MR, Kambampati V, Mains RE, Eipper BA (2000) An isoform of kalirin, a brain-specific GDP/GTP exchange factor, is enriched in the postsynaptic density fraction. J Biol Chem 275:63956403. CrossRef Medline

Penzes P, Johnson RC, Sattler R, Zhang X, Huganir RL, Kambampati V, Mains RE, Eipper BA (2001) The neuronal Rho-GEF Kalirin-7 interacts with PDZ domain-containing proteins and regulates dendritic morphogenesis. Neuron 29:229-242. CrossRef Medline

Penzes P, Beeser A, Chernoff J, Schiller MR, Eipper BA, Mains RE, Huganir RL (2003) Rapid induction of dendritic spine morphogenesis by transsynaptic ephrinB-EphB receptor activation of the Rho-GEF kalirin. Neuron 37:263-274. CrossRef Medline

Petralia RS, Sans N, Wang YX, Wenthold RJ (2005) Ontogeny of postsynaptic density proteins at glutamatergic synapses. Mol Cell Neurosci 29: 436-452. CrossRef Medline

Phillips MA, Colonnese MT, Goldberg J, Lewis LD, Brown EN, ConstantinePaton M (2011) A synaptic strategy for consolidation of convergent visuotopic maps. Neuron 71:710-724. CrossRef Medline 
Ramakers GJ (2002) Rho proteins, mental retardation and the cellular basis of cognition. Trends Neurosci 25:191-199. CrossRef Medline

Rauner C, Köhr G (2011) Triheteromeric NR1/NR2A/NR2B receptors constitute the major $\mathrm{N}$-methyl-D-aspartate receptor population in adult hippocampal synapses. J Biol Chem 286:7558-7566. CrossRef Medline

Rejeb I, Saillour Y, Castelnau L, Julien C, Bienvenu T, Taga P, Chaabouni H, Chelly J, Ben Jemaa L, Bahi-Buisson N (2008) A novel splice mutation in PAK3 gene underlying mental retardation with neuropsychiatric features. Eur J Hum Genet 16:1358-1363. CrossRef Medline

Ropers HH (2010) Genetics of early onset cognitive impairment. Annu Rev Genomics Hum Genet 11:161-187. CrossRef Medline

Rubinson DA, Dillon CP, Kwiatkowski AV, Sievers C, Yang L, Kopinja J, Rooney DL, Zhang M, Ihrig MM, McManus MT, Gertler FB, Scott ML, Van Parijs L (2003) A lentivirus-based system to functionally silence genes in primary mammalian cells, stem cells and transgenic mice by RNA interference. Nat Genet 33:401-406. CrossRef Medline

Saneyoshi T, Wayman G, Fortin D, Davare M, Hoshi N, Nozaki N, Natsume T, Soderling TR (2008) Activity-dependent synaptogenesis: regulation by a CaM-kinase kinase/CaM-kinase I/betaPIX signaling complex. Neuron 57:94-107. CrossRef Medline

Sans N, Petralia RS, Wang YX, Blahos J 2nd, Hell JW, Wenthold RJ (2000) A developmental change in NMDA receptor-associated proteins at hippocampal synapses. J Neurosci 20:1260-1271. Medline

Scherer SW, Dawson G (2011) Risk factors for autism: translating genomic discoveries into diagnostics. Hum Genet 130:123-148. CrossRef Medline

Schlüter OM, Xu W, Malenka RC (2006) Alternative N-terminal domains of PSD-95 and SAP97 govern activity-dependent regulation of synaptic AMPA receptor function. Neuron 51:99-111. CrossRef Medline

Sheng M, Hoogenraad CC (2007) The postsynaptic architecture of excitatory synapses: a more quantitative view. Annu Rev Biochem 76:823-847. CrossRef Medline

Takasu MA, Dalva MB, Zigmond RE, Greenberg ME (2002) Modulation of NMDA receptor-dependent calcium influx and gene expression through EphB receptors. Science 295:491-495. CrossRef Medline

Tarpey P, Parnau J, Blow M, Woffendin H, Bignell G, Cox C, Cox J, Davies H, Edkins S, Holden S, Korny A, Mallya U, Moon J, O’Meara S, Parker A, Stephens P, Stevens C, Teague J, Donnelly A, Mangelsdorf M, et al. (2004) Mutations in the DLG3 gene cause nonsyndromic X-linked mental retardation. Am J Hum Genet 75:318-324. CrossRef Medline
Tolias KF, Bikoff JB, Kane CG, Tolias CS, Hu L, Greenberg ME (2007) The Racl guanine nucleotide exchange factor Tiam1 mediates EphB receptordependent dendritic spine development. Proc Natl Acad Sci U S A 104: 7265-7270. CrossRef Medline

van Zundert B, Yoshii A, Constantine-Paton M (2004) Receptor compartmentalization and trafficking at glutamate synapses: a developmental proposal. Trends Neurosci 27:428-437. CrossRef Medline

Washbourne P, Liu XB, Jones EG, McAllister AK (2004) Cycling of NMDA receptors during trafficking in neurons before synapse formation. J Neurosci 24:8253-8264. CrossRef Medline

Xie Z, Srivastava DP, Photowala H, Kai L, Cahill ME, Woolfrey KM, Shum CY, Surmeier DJ, Penzes P (2007) Kalirin-7 controls activity-dependent structural and functional plasticity of dendritic spines. Neuron 56:640-656. CrossRef Medline

Yoshii A, Constantine-Paton M (2007) BDNF induces transport of PSD-95 to dendrites through PI3K-AKT signaling after NMDA receptor activation. Nat Neurosci 10:702-711. CrossRef Medline

Yoshii A, Sheng MH, Constantine-Paton M (2003) Eye opening induces a rapid dendritic localization of PSD-95 in central visual neurons. Proc Natl Acad Sci U S A 100:1334-1339. CrossRef Medline

Yoshii A, Murata Y, Kim J, Zhang C, Shokat KM, Constantine-Paton M (2011) TrkB and protein kinase M \{zeta\} regulate synaptic localization of PSD-95 in developing cortex. J Neurosci 31:11894-11904. CrossRef Medline

Zanni G, van Esch H, Bensalem A, Saillour Y, Poirier K, Castelnau L, Ropers HH, de Brouwer AP, Laumonnier F, Fryns JP, Chelly J (2010) A novel mutation in the DLG3 gene encoding the synapse-associated protein 102 (SAP102) causes nonsyndromic mental retardation. Neurogenetics 11: 251-255. CrossRef Medline

Zhang H, Webb DJ, Asmussen H, Niu S, Horwitz AF (2005) A GIT1/PIX/ Rac/PAK signaling module regulates spine morphogenesis and synapse formation through MLC. J Neurosci 25:3379-3388. CrossRef Medline

Zheng CY, Petralia RS, Wang YX, Kachar B, Wenthold RJ (2010) SAP102 is a highly mobile MAGUK in spines. J Neurosci 30:4757-4766. CrossRef Medline

Zheng CY, Seabold GK, Horak M, Petralia RS (2011) MAGUKs, synaptic development, and synaptic plasticity. Neuroscientist 17:493-512. CrossRef Medline 